\title{
Relative fixed point theory
}

\author{
Kate Ponto
}

\begin{abstract}
The Lefschetz fixed point theorem and its converse have many generalizations. One of these generalizations is to endomorphisms of a space relative to a fixed subspace. In this paper we define relative Lefschetz numbers and Reidemeister traces using traces in bicategories with shadows. We use the functoriality of this trace to identify different forms of these invariants and to prove a relative Lefschetz fixed point theorem and its converse.
\end{abstract}

55M20; 18D05, 55P25

\section{Introduction}

The goal of topological fixed point theory is to find invariants that detect if a given endomorphism of a space has any fixed points. The Lefschetz fixed point theorem identifies one such invariant.

Theorem (Lefschetz fixed point theorem) Let $B$ be a closed smooth manifold and

$$
f: B \rightarrow B
$$

be a continuous map. If $f$ has no fixed points then the Lefschetz number of $f$,

$$
L(f):=\sum_{i}(-1)^{i} \operatorname{tr}\left(H_{i}(f ; \mathbb{Q})\right),
$$

is zero.

This gives a necessary, but usually not sufficient, condition for determining if a continuous map does not have any fixed points.

If we put additional restrictions on the map $f$, such as requiring it to preserve a subspace of $B$, the Lefschetz number still gives a necessary condition for $f$ to be fixed point free. However, this invariant ignores the relative structure and so is not the best possible invariant. For example, the identity map of the circle is homotopic to a map with no fixed points and so the Lefschetz number is zero. If this map is required to 
preserve a nonempty, proper subinterval it is no longer homotopic to a map with no fixed points.

There is a refinement of the Lefschetz number defined using the induced maps on the rational homology of the subspace and the relative rational homology.

Theorem A (Relative Lefschetz fixed point theorem) Let $A \subset B$ be closed smooth manifolds and

$$
f: B \rightarrow B
$$

be a continuous map such that $f(A) \subset A$. If $f$ has no fixed points then the relative Lefschetz number of $f$ is zero.

The relative Lefschetz number of the identity map of the circle relative to a nonempty, proper subinterval is not zero.

Both of these theorems give a condition that implies that a continuous endomorphism

$$
f: B \rightarrow B
$$

has a fixed point. In most cases they do not give a condition that implies $f$ has no fixed points. To address this question a refined invariant and some restrictions on the spaces have to be introduced. This refined invariant is called the Nielsen number or the Reidemeister trace.

Theorem (Converse to the Lefschetz fixed point theorem) Let $B$ be a closed smooth manifold of dimension at least 3 and

$$
f: B \rightarrow B
$$

be a continuous map. The map $f$ is homotopic to a map with no fixed points if and only if the Reidemeister trace of $f$ is zero.

The Reidemeister trace is a partitioning of the Lefschetz number to reflect the ways fixed points can be changed by a homotopy of the original map. There is a generalization of the Reidemeister trace to a relative Reidemeister trace that is very similar to the generalization of the Lefschetz number to the relative Lefschetz number.

Theorem B (Converse to the relative Lefschetz fixed point theorem) Suppose $A \subset B$ are closed smooth manifolds of dimension at least 3 and the codimension of $A$ in $B$ is at least 2. A continuous map

$$
f: B \rightarrow B
$$

such that $f(A) \subset A$ is homotopic to a map with no fixed points via a homotopy $H$ satisfying $H(A, t) \subset A$ if and only if the relative Reidemeister trace of $f$ is zero. 
The goal of this paper is to provide definitions of the relative Lefschetz number and relative Reidemeister trace and proofs of Theorems A and B that satisfy several requirements. First, the relative Reidemeister trace should detect if a map is relatively homotopic to a map with no fixed points. It is not necessary for the relative Reidemeister trace to provide a lower bound for the number of fixed points. Second, the invariants should satisfy cyclicity and naturality conditions associated with the trace in linear algebra. The relative Reidemeister trace should to be compatible with the approach of Klein and Williams in $[14 ; 15]$. Those papers give a proof of the converse to the Lefschetz fixed point theorem that is different from the standard simplicial proof. Finally, the relative Reidemeister trace should be compatible with an equivariant generalization of the Reidemeister trace described in Ponto [20].

While the Lefschetz number and the Reidemeister trace have long established definitions, the relative forms of these invariants are less settled. Versions of the relative Lefschetz number have been defined by Bowszyc [1] and Jezierski [11] and of the relative Reidemeister trace by Norton-Odenthal and Wong [19], Schirmer [24] and Zhao [30; 31; 32]. The invariants of Schirmer [24] and Zhao [30; 31] are primarily interested in determining lower bounds for the number of fixed points and so are generalizations of the Nielsen number. The invariants defined by Norton-Odenthal and Wong [19] and Zhao [32] have more of the properties associated with traces, but the definition are still motivated by connections to the Nielsen number. None of these invariants satisfy all of our conditions above, and none of them exactly coincide with the definitions given here. In this paper we give proofs of Theorems A and B following the outline of Ponto [21]. We use duality and trace in bicategories with shadows to define two forms of the relative Lefschetz number and the relative Reidemeister trace. Then, using functoriality, we show these invariants coincide. Finally, we generalize Klein and Williams' proof of the converse to the Lefschetz fixed point theorem in [14] to complete the proof of the converse to the relative Lefschetz fixed point theorem.

In the first two sections we will recall the necessary definitions of duality and trace in symmetric monoidal categories and in bicategories with shadows. In Section 3 we will describe some examples of bicategories with shadows and generalize results from Ponto [21] that describe specific examples of duals.

In Section 4 we apply this category theory to the relative Lefschetz number. In Sections 5 and 6 we define the relative Reidemeister trace. We describe how to compare these invariants to each other and how to compare them to the relative Nielsen number defined by Schirmer [24] and Zhao [30]. In Section 7 and Section 8 we give a proof of the converse to the relative Lefschetz fixed point theorem following the proofs given by Klein and Williams in $[14 ; 15]$. In Section 9 we include some formal results omitted from the third section. 
Acknowledgments The author would like to thank Peter May, Gun Sunyeekhan and Bruce Williams for many helpful conversations and comments on previous drafts of this paper. She would also like to thank Xue Zhi Zhao for providing a preprint.

The author was partially supported by a NSF postdoctoral fellowship.

\section{A review of Nielsen theory}

In this section let $B$ be a closed smooth manifold or, more generally, a compact ENR. Let $f: B \rightarrow B$ be a continuous map.

Let $U \subset B$ be an open subset so that $f$ has no fixed points on $\partial U$. Since $B$ is an ENR, there is an open subset $W$ of $\mathbb{R}^{n}$, containing $B$, and a retraction $r: W \rightarrow B$. Let $V=r^{-1}(U)$ and $F_{U}$ be the fixed points of $\left.f\right|_{U}$.

Let $I(f, U)$ be the composite

$$
\begin{aligned}
H^{n}\left(S^{n}\right) \leftarrow H^{n}\left(S^{n}, S^{n} \backslash 0\right) \rightarrow & H^{n}\left(\mathbb{R}^{n}, \mathbb{R}^{n} \backslash 0\right) \\
& H^{n}\left(V, \stackrel{V}{V} \backslash F_{U}\right)<H^{n}\left(S^{n}, S^{n} \backslash F_{U}\right) \rightarrow H^{n}\left(S^{n}\right) .
\end{aligned}
$$

This is homology with rational coefficients. The horizontal maps are given by excision or the long exact sequence of a pair. The maps that point to the left are isomorphisms. The vertical map is induced by $x \mapsto x-f r(x)$. If $\left[S^{n}\right]$ is a generator of $H^{n}\left(S^{n}\right)$, let $i(f, U)$ be the rational number defined by

$$
I(f, U)\left(\left[S^{n}\right]\right)=i(f, U)\left[S^{n}\right] .
$$

If $U=B, i(f, B)$ is denoted $i(f)$ and is called the fixed point index of $f$. If $F$ is a set of fixed points of $f$ and $U \subset B$ is an open set containing $F$ such that $\operatorname{Fix}\left(\left.f\right|_{\bar{U}}\right)=F$, $i(f, U)$ is denoted $i(f, F)$ and is called the fixed point index of $F$. This independent of the choice of $U$. For more details see Dold [6].

If $f$ is homotopic to a map with no fixed points $i(f)$ is zero.

Theorem 0.1 (Lefschetz-Hopf) $L(f)=i(f)$.

The Lefschetz fixed point theorem is a corollary of this result. We will describe a proof of Theorem 0.1 in Section 1.

For the converse to the Lefschetz fixed point theorem we need to refine the Lefschetz number and index. Let $\Lambda^{f} B:=\left\{\gamma \in B^{I} \mid f(\gamma(0))=\gamma(1)\right\}$. Constant paths define a function $\psi$ from the fixed points of $f$ into $\pi_{0}\left(\Lambda^{f} B\right)$. Two fixed points of $f$ are in 
the same fixed point class if they have the same image under $\psi$. This agrees with the standard definition of fixed point classes in Brown [2] or Jiang [12]. The (geometric) Reidemeister trace of $f$ is

$$
R^{\mathrm{ge}}(f):=\sum_{\text {fixed point classes } \alpha} i(f, \alpha)[\alpha] \in \mathbb{Q} \pi_{0}\left(\Lambda^{f} B\right) .
$$

Here we identify an element $\alpha \in \Lambda^{f} B$ with the fixed points in the associated fixed point class. The Nielsen number of $f, N(f)$, is the number of fixed point classes $\alpha$ with $i(f, \alpha) \neq 0$.

There is also a generalization of the Lefschetz number. The action of the fundamental group $\pi_{1}(B)$ on the universal cover of $B, \widetilde{B}$, induces an action of $\mathbb{Q} \pi_{1}(B)$ on $C_{*}(\widetilde{B} ; \mathbb{Q})$. The endomorphism $f$ induces endomorphisms

$$
\phi: \pi_{1}(B) \rightarrow \pi_{1}(B) \quad \text { and } \quad f_{*}: C_{*}(\widetilde{B} ; \mathbb{Q}) \rightarrow C_{*}(\widetilde{B} ; \mathbb{Q}) .
$$

If $\alpha \in \pi_{1}(B)$ and $\gamma \in C_{*}(\widetilde{B} ; \mathbb{Q})$ these maps satisfy $f_{*}(\gamma \alpha)=f_{*}(\gamma) \phi(\alpha)$. The Hattori-Stallings trace of $f_{*}$ is called the generalized Lefschetz number or (global) Reidemeister trace of $f$ and is denoted $R^{\mathrm{gl}}(f)$.

The following identification of invariants, generalizing Theorem 0.1, is due to Husseini [9].

Theorem $0.2\left[9\right.$, Theorem 1.13] $R^{\mathrm{ge}}(f)=R^{\mathrm{gl}}(f)$.

The following converse to the Lefschetz fixed point theorem is due to Reidemeister [23] and Wecken [28].

Theorem 0.3 (Converse to the Lefschetz fixed point theorem) Assume $B$ is a closed smooth manifold of dimension at least 3. Then $f$ is homotopic to a map with $N(f)$ fixed points.

These two theorems give a proof of the converse to the Lefschetz fixed point theorem in the introduction.

\section{Preliminaries on cofibrations}

We fix some conventions and recall a fact about cofibrations. Let $A \subset B$ and $X \subset Y$ be topological spaces.

Definition 0.4 A map $f: B \rightarrow Y$ is a relative map if $f(A) \subset X$. 
We will write this

$$
f:(B, A) \rightarrow(Y, X) .
$$

A homotopy $H: B \times I \rightarrow Y$ is a relative homotopy if $\left.H\right|_{A \times I}$ factors through the inclusion $X \subset Y$.

The following definition and lemma are standard in relative fixed point theory. See, for example, Zhao [30].

Definition 0.5 [30, Definition 3.1] A relative map $f:(B, A) \rightarrow(B, A)$ is taut if there is a neighborhood $N(A)$ of $A$ in $B$ such that $f(N(A)) \subset A$.

We will use this condition to isolate the fixed points of $A$ from those of $B \backslash A$.

Lemma 0.6 [30, Lemma 3.2] If $A \subset B$ is a cofibration then any relative map $f:(B, A) \rightarrow(B, A)$ is relatively homotopic to a taut map.

In this paper we will assume $A \subset B$ is a cofibration and all relative maps

$$
f:(B, A) \rightarrow(B, A) .
$$

are taut. If a relative map is not taut it is implicitly replaced by a relatively homotopic map that is taut. Since all invariants defined here are invariants of the relative homotopy class, the choice of replacement does not matter.

\section{Duality and trace in symmetric monoidal categories}

Duality and trace in symmetric monoidal categories is a generalization of the trace in linear algebra that retains many important properties. The trace in a symmetric monoidal category satisfies a generalization of invariance of basis and is functorial. The Lefschetz fixed point theorem is one application of the functoriality of the trace. This section is a very brief summary of Dold and Puppe's results in [7]. For more details see [7], Lewis, May and Steinberger [16, Chapter III] or Ponto and Shulman [22].

Let $\mathscr{C}$ be a symmetric monoidal category with monoidal product $\otimes$, unit $S$, and symmetry isomorphism

$$
\gamma: X \otimes Y \rightarrow Y \otimes X
$$

Definition 1.1 An object $A$ in $\mathscr{C}$ is dualizable with dual $B$ if there are maps

$$
\eta: S \rightarrow A \otimes B
$$

and

$$
\epsilon: B \otimes A \rightarrow S
$$


such that the composites

and

$$
A \cong S \otimes A \stackrel{\eta \otimes \mathrm{id}}{\longrightarrow} A \otimes B \otimes A \stackrel{\text { id } \otimes \epsilon}{\longrightarrow} A \otimes S \cong A
$$

$$
B \cong B \otimes S \stackrel{\mathrm{id} \otimes \eta}{\longrightarrow} B \otimes A \otimes B \stackrel{\epsilon \otimes \mathrm{id}}{\longrightarrow} S \otimes B \cong B
$$

are the identity maps of $A$ and $B$ respectively.

A familiar example of a symmetric monoidal category is the category of modules over a commutative ring $R$. The tensor product is the monoidal product. If $M$ is a finitely generated projective $R$-module, $M$ is dualizable and the dual of $M$ is $\operatorname{Hom}_{R}(M, R)$. The evaluation map

$$
\epsilon: \operatorname{Hom}_{R}(M, R) \otimes_{R} M \rightarrow R
$$

is defined by $\epsilon(\phi, m)=\phi(m)$. Since $M$ is finitely generated and projective the dual basis theorem implies there is a "basis" $\left\{m_{1}, m_{2}, \ldots, m_{n}\right\}$ with dual "basis" $\left\{m_{1}^{\prime}, m_{2}^{\prime}, \ldots, m_{n}^{\prime}\right\}$. The coevaluation is given by linearly extending

$$
\eta(1)=\sum m_{i} \otimes m_{i}^{\prime}
$$

The category of chain complexes of modules over a commutative ring $R$ is also symmetric monoidal. The dualizable objects are the chain complexes that are projective in each degree and finitely generated.

Definition 1.2 If $A$ is dualizable and $f: A \rightarrow A$ is an endomorphism in $\mathscr{C}$, the trace of $f, \operatorname{tr}(f)$, is the composite

$$
S \stackrel{\eta}{\longrightarrow} A \otimes B \stackrel{f \otimes \mathrm{id}}{\longrightarrow} A \otimes B \stackrel{\gamma}{\longrightarrow} B \otimes A \stackrel{\epsilon}{\longrightarrow} S .
$$

The trace of an endomorphism in the symmetric monoidal category of vector spaces over a field is the sum of the diagonal elements in a matrix representation. The trace of an endomorphism in the category of chain complexes of modules over a commutative ring is the Lefschetz number. It is the alternating sum of the level wise traces.

Proposition 1.3 Let $F: \mathscr{C} \rightarrow \mathscr{D}$ be a symmetric monoidal functor, $A$ be a dualizable object of $\mathscr{C}$ with dual $B$, and

$$
F(A) \otimes F(B) \rightarrow F(A \otimes B)
$$

and

$$
S_{\mathscr{D}} \rightarrow F\left(S_{\mathscr{C}}\right)
$$

be isomorphisms. Then $F(A)$ is dualizable with dual $F(B)$. If $f: A \rightarrow A$ is an endomorphism in $\mathscr{C}, F(\operatorname{tr}(f))=\operatorname{tr}(F(f))$. 
The stable homotopy category is a symmetric monoidal category. There is also a way to express duality for spaces without using spectra.

Definition 1.4 A compact based space $X$ is $n$-dualizable if there is a compact based space $Y$ and continuous maps $\eta: S^{n} \rightarrow X \wedge Y$ and $\epsilon: Y \wedge X \rightarrow S^{n}$ such that the diagrams
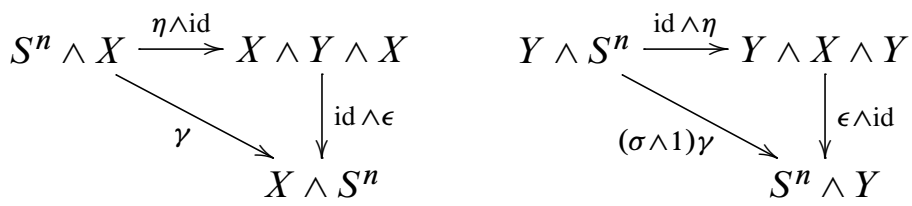

commute up to stable homotopy.

The map $\sigma: S^{n} \rightarrow S^{n}$ is defined by $\sigma(v)=-v$.

The following characterizations of dualizable spaces can be found in Lewis, May and Steinberger [16] and May and Sigurdsson [18].

Proposition 1.5 [16, Theorem III.4.1, Theorem III.5.1; 18, Theorem 18.6.5]

(1) If $M$ is a closed smooth manifold that embeds in $\mathbb{R}^{m}$, then $M_{+}$is dualizable with dual $T v$, the Thom space of the normal bundle of the embedding of $M$ in $\mathbb{R}^{m}$.

(2) If $L$ is a closed smooth submanifold of a closed smooth manifold $M$ that embeds in $\mathbb{R}^{m}$, then $M \cup C L$ is dualizable with dual $T v_{M} \cup C T v_{L}$.

(3) If $B$ is a compact ENR that embeds in $\mathbb{R}^{n}, B_{+}$is dualizable with dual the cone on the inclusion $\mathbb{R}^{n} \backslash B \rightarrow \mathbb{R}^{n}$.

(4) If $B$ is a compact ENR that embeds in $\mathbb{R}^{n}$ and $A$ is a sub-ENR of $B$, then $B \cup C A$ is dualizable with dual $\left(\mathbb{R}^{n} \backslash A\right) \cup C\left(\mathbb{R}^{n} \backslash B\right)$.

Here $C$ denotes the cone. If $A \subset B$ then $B \cup C A$ is the mapping cone on the inclusion $A \rightarrow B$. The base point of $B \cup C A$ is the cone point.

The trace of an endomorphism of spaces regarded as a map in the stable homotopy category is the fixed point index. The index is the stable homotopy class of a map

$$
S^{n} \rightarrow S^{n}
$$

and so is an element of the 0 -th stable homotopy group of $S^{0}, \pi_{0}^{s}$. This agrees with the definition of the index in the introduction.

Since the rational homology functor is strong symmetric monoidal, Proposition 1.3 implies that the fixed point index of a map $f$ is equal to the Lefschetz number of $H_{*}(f)$, Theorem 0.1 . 


\section{Duality and trace for bicategories with shadows}

Unfortunately, the Reidemeister trace cannot be defined as a trace in a symmetric monoidal category. It can be defined using the more general trace in a bicategory with shadows. Duality and trace in a bicategory are very similar to duality and trace in a symmetric monoidal category but are more flexible. This is a brief summary of May and Sigurdsson [18, Chapter 16] and Ponto [21, Chapter 4]. For more details see either of those references or Ponto and Shulman [22].

Definition 2.1 A bicategory $\mathscr{B}$ consists of

(1) a collection ob $\mathscr{B}$,

(2) categories $\mathscr{B}(A, B)$ for each $A, B \in$ ob $\mathscr{B}$,

(3) functors

$$
\begin{gathered}
\odot: \mathscr{B}(B, C) \times \mathscr{B}(A, B) \rightarrow \mathscr{B}(A, C) \\
U_{A}: * \rightarrow \mathscr{B}(A, A)
\end{gathered}
$$

for $A, B$ and $C$ in ob $\mathscr{B}$.

Here $*$ denotes the category with one object and one morphism. The functors $\odot$ are required to satisfy unit and associativity conditions.

The elements of ob $\mathscr{B}$ are called 0 -cells. The objects of $\mathscr{B}(A, B)$ are called 1-cells. The morphisms of $\mathscr{B}(A, B)$ are called 2-cells.

A familiar example of a bicategory is the bicategory Mod with 0 -cells rings, 1-cells bimodules, and 2-cells homomorphisms. The bicategory composition is tensor product.

Definition 2.2 [18, Definition 16.4.1] A 1-cell $X \in \mathscr{B}(B, A)$ is right dualizable with dual $Y \in \mathscr{B}(A, B)$ if there are 2-cells

$$
\eta: U_{A} \rightarrow X \odot Y, \quad \epsilon: Y \odot X \rightarrow U_{B}
$$

such that

$$
\begin{aligned}
& X \cong U_{A} \odot X \stackrel{\eta \odot \text { id }}{\longrightarrow} X \odot Y \odot X \stackrel{\text { id } \odot \epsilon}{\longrightarrow} X \odot U_{B} \cong X \\
& Y \cong Y \odot U_{A} \stackrel{\text { id } \odot \eta}{\longrightarrow} Y \odot X \odot Y \stackrel{\epsilon \odot \text { id }}{\longrightarrow} U_{B} \odot Y \cong Y
\end{aligned}
$$

are the identity maps of $X$ and $Y$ respectively. 
The map $\eta$ is called the coevaluation and $\epsilon$ is called the evaluation.

If $R$ is a (not necessarily commutative) ring and $M$ is a finitely generated projective right $R$-module then $M$ is a right dualizable 1 -cell in $\operatorname{Mod}$ with dual $\operatorname{Hom}_{R}(M, R)$. The evaluation map

$$
\epsilon: \operatorname{Hom}_{R}(M, R) \otimes_{\mathbb{Z}} M \rightarrow R
$$

is defined by $\epsilon(\phi, m)=\phi(m)$. This is a map of $R-R$-bimodules. Since $M$ is finitely generated and projective there are elements $\left\{m_{1}, m_{2}, \ldots, m_{n}\right\}$ and dual elements $\left\{m_{1}^{\prime}, m_{2}^{\prime}, \ldots, m_{n}^{\prime}\right\}$ of $\operatorname{Hom}_{R}(M, R)$ so that the coevaluation map

$$
\eta: \mathbb{Z} \rightarrow M \otimes_{R} \operatorname{Hom}_{R}(M, R)
$$

is defined by linearly extending $\eta(1)=\sum m_{i} \otimes m_{i}^{\prime}$. This is a map of abelian groups.

Unlike the symmetric monoidal case, we need more structure before we can define the trace. The additional structure is a shadow.

Definition 2.3 [21, Definition 4.4.1] A shadow for $\mathscr{B}$ is a functor

$$
\left\langle\langle-\rangle: \coprod_{A \in \mathrm{ob} \Re} \mathscr{B}(A, A) \rightarrow \mathscr{T}\right.
$$

to a category $\mathscr{T}$ and a natural isomorphism

$$
\theta:\langle\langle X \odot Y\rangle\rangle \cong\langle\langle Y \odot X\rangle\rangle
$$

for every pair of 1 -cells $X \in \mathscr{B}(A, B)$ and $Y \in \mathscr{B}(B, A)$ such that the diagrams
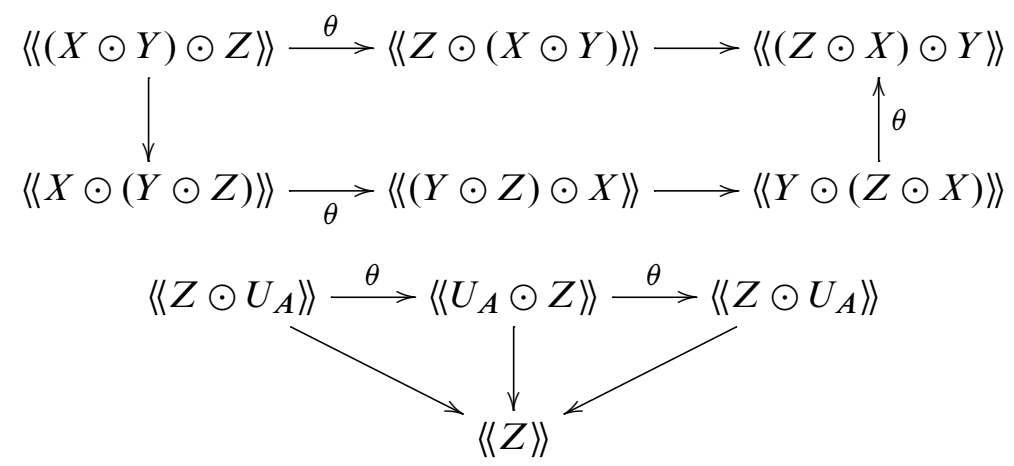

commute whenever they are defined.

Let $P$ be an $R-R$-bimodule. Let $N(P)$ be the subgroup of $P$ generated by elements of the form

$$
r p-p r
$$

for $p \in P$ and $r \in R$. Then the shadow of $P$ is $P / N(P)$. 
Definition 2.4 [21, Definition 4.5.1] Let $X$ be a dualizable 1-cell and $f: Q \odot X \rightarrow$ $X \odot P$ be a 2 -cell in $\mathscr{B}$. The trace of $f$ is the composite

$$
\begin{aligned}
\left\langle\langle Q\rangle \cong\left\langle\left\langle Q \odot U_{A}\right\rangle\right\rangle \stackrel{\text { id } \odot \eta}{\longrightarrow}\right. & \langle\langle Q \odot X \odot Y\rangle\rangle \\
& \quad \mid f \odot \text { id } \\
& \langle\langle X \odot P \odot Y\rangle\rangle \stackrel{\theta}{\longrightarrow}\langle\langle P \odot Y \odot X\rangle) \stackrel{\text { id } \odot \epsilon}{\longrightarrow}\left\langle\left\langle P \odot U_{B}\right\rangle \cong\langle\langle P\rangle\rangle .\right.
\end{aligned}
$$

If $M$ is a finitely generated projective right $R$-module and $f: M \rightarrow M$ is a map of right $R$-modules the trace of $f$ is the trace defined by Stallings in [26, Section 1.7].

A shadow functor is a functor of bicategories $F$ and a natural transformation

such that

$$
\psi:\langle\langle F(-)\rangle\rangle \rightarrow F(\langle\langle-\rangle)
$$

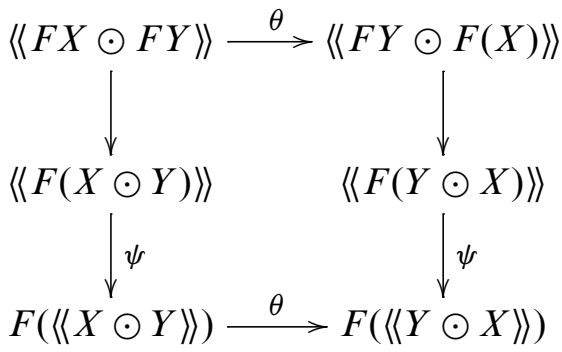

commutes for all 1-cells $X$ and $Y$ where $X \odot Y$ and $Y \odot X$ are both defined.

Proposition 2.5 [21, Proposition 4.5.7] Let $X$ be a right dualizable 1-cell in $\mathscr{B}$ with dual $Y$,

$$
f: Q \odot X \rightarrow X \odot P
$$

be a 2-cell in $\mathscr{B}$ and $F: \mathscr{B} \rightarrow \mathscr{B}^{\prime}$ be a shadow functor. If $F(X) \odot F(Y) \rightarrow F(X \odot Y)$, $F(X) \odot F(P) \rightarrow F(X \odot P)$, and $U_{F(B)} \rightarrow F\left(U_{B}\right)$ are isomorphisms and $\hat{f}$ is the composite

$$
F Q \odot F X \stackrel{\phi}{\longrightarrow} F(Q \odot X) \stackrel{F(f)}{\longrightarrow} F(X \odot P) \stackrel{\phi^{-1}}{\longrightarrow} F X \odot F P
$$

then

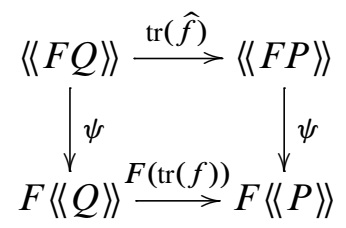

commutes. 
We will use this proposition to compare different forms of the Lefschetz number and Reidemeister trace.

\section{Some examples of bicategories with shadows}

The classical descriptions of fixed point invariants require a choice of base point. When working with a single space this isn't a problem. With fiberwise spaces, equivariant spaces, or pairs of spaces, choosing base points requires addition conditions on the space.

In this section we describe a generalization of the bicategory Mod that we will use to define fixed point invariants without choosing base points. In this bicategory we replace rings by categories, modules by functors, and homomorphisms by natural transformations.

Let $\mathscr{V}$ be a symmetric monoidal category with monoidal product $\otimes$ and unit $S$.

Definition 3.1 A category $\mathscr{A}$ is enriched in $\mathscr{V}$ if for each $a, b \in \mathrm{ob}(\mathscr{A}), \mathscr{A}(a, b)$ is an object of $\mathscr{V}$ and the composition for $\mathscr{A}$,

$$
\mathscr{A}(b, c) \otimes \mathscr{A}(a, b) \rightarrow \mathscr{A}(a, c),
$$

is a morphism in $\mathscr{V}$.

For pairs of enriched categories $\mathscr{A}$ and $\mathscr{B}$ define an enriched category $\mathscr{A} \otimes \mathscr{B}$ with objects pairs $(a, b)$ where $a \in \mathrm{ob} \mathscr{A}$ and $b \in \mathrm{ob} \mathscr{B}$. If $a, a^{\prime} \in \mathrm{ob} \mathscr{A}$ and $b, b^{\prime} \in \mathrm{ob} \mathscr{B}$, then

$$
(\mathscr{A} \otimes \mathscr{B})\left((a, b),\left(a^{\prime}, b^{\prime}\right)\right)=\left(\mathscr{A}\left(a, a^{\prime}\right)\right) \otimes\left(\mathscr{B}\left(b, b^{\prime}\right)\right) .
$$

Definition 3.2 An enriched distributor is a functor $\mathscr{X}: \mathscr{A} \otimes \mathscr{B}^{\mathrm{op}} \rightarrow \mathscr{V}$ such that the actions of morphisms of $\mathscr{A}$ and $\mathscr{B}$ on $\mathscr{X}$ are maps in $\mathscr{V}$.

This type of functor is also called an $\mathscr{A}$ - $\mathscr{B}$-bimodule. If $F: \mathscr{A} \rightarrow \mathscr{C}$ is an enriched functor and $\mathscr{Y}: \mathscr{C} \otimes \mathscr{B}^{\mathrm{op}} \rightarrow \mathscr{V}$ is a distributor define a new distributor $\mathscr{Y}^{F}: \mathscr{A} \otimes \mathscr{B}^{\mathrm{op}} \rightarrow \mathscr{V}$ by $y^{F}(a, b)=y(F(a), b)$.

Definition 3.3 An enriched natural transformation $\eta: \mathscr{X} \rightarrow \mathscr{Y}$ is a natural transformation where the maps

$$
\eta_{a, b}: \mathscr{X}(a, b) \rightarrow \mathscr{Y}(a, b)
$$

are maps in $\mathscr{V}$ for all $a \in \mathrm{ob} \mathscr{A}$ and $b \in \mathrm{ob} \mathscr{B}$. 
Enriched categories are the 0 -cells of a bicategory we denote by $\mathscr{E} q$. The 1 -cells are the distributors enriched in $\mathscr{V}$. The 2-cells are enriched natural transformations. If $\mathscr{X}: \mathscr{A} \otimes \mathscr{B}^{\mathrm{op}} \rightarrow \mathscr{V}$ and $\mathscr{Y}: \mathscr{B} \otimes \mathscr{C}^{\mathrm{op}} \rightarrow \mathscr{V}$ are two distributors, $\mathscr{X} \odot \mathscr{Y}$ is a distributor $\mathscr{A} \otimes \mathscr{C}^{\mathrm{op}} \rightarrow \mathscr{V}$. For $a \in \mathrm{ob}(\mathscr{A})$ and $c \in \mathrm{ob}(\mathscr{C}),(\mathscr{L} \odot \mathscr{Y})(a, c)$ is the coequalizer of the actions of $\mathscr{B}$ on $\mathscr{X}$ and $\mathscr{Y}$,

$$
\coprod_{b, b^{\prime} \in \mathrm{ob} \mathscr{B}} \mathscr{X}(a, b) \otimes \mathscr{B}\left(b^{\prime}, b\right) \otimes \mathscr{Y}\left(b^{\prime}, c\right) \Longrightarrow \coprod_{b \in \mathrm{ob} \mathscr{B}} \mathscr{X}(a, b) \otimes \mathscr{Y}(b, c) .
$$

If $\mathscr{L}: \mathscr{A} \otimes \mathscr{A}^{\mathrm{op}} \rightarrow \mathscr{V}$ is an enriched functor, the shadow of $\mathscr{L},\langle\langle\mathscr{L}\rangle\rangle$, is the coequalizer of the two actions of $\mathscr{A}$ on $\mathscr{L}$,

$$
\coprod_{a, a^{\prime} \in \mathrm{ob}(\mathscr{A})} \mathscr{A}\left(a, a^{\prime}\right) \otimes \mathscr{L}\left(a, a^{\prime}\right) \Longrightarrow \coprod_{a \in \mathrm{ob}(\mathscr{A})} \mathscr{L}(a, a) .
$$

In [21, Chapter 9] we observed that if $\mathscr{A}$ is a connected groupoid, a distributor

$$
\mathscr{L}: \mathscr{A} \rightarrow \mathscr{V}
$$

is dualizable if and only if $\mathscr{X}(a)$ is dualizable over $\mathscr{A}(a, a)$ for any $a \in \operatorname{ob} \mathscr{A}$. The categories we will use here and in Ponto [20] to define relative and equivariant fixed point invariants are not usually groupoids, but we can extend the results from Ponto [21] to describe these examples.

We begin by recalling a definition from Lück [17].

Definition 3.4 [17, Definition II.9.2] A category $\mathscr{A}$ is an EI-category if all endomorphisms are isomorphisms.

In an EI-category $\mathscr{A}$ there is a partial order on the set of objects: $x<y$ if $\mathscr{A}(x, y) \neq \varnothing$.

Let $\mathrm{Ch}_{R}$ be the symmetric monoidal category of chain complexes of modules over a commutative ring $R$ and chain maps. Let $\mathscr{A}$ be a category enriched in the category of modules over $R$. This can be regarded as a category enriched in chain complexes concentrated in degree zero.

Definition 3.5 A functor $\mathscr{X}: \mathscr{A} \rightarrow \mathrm{Ch}_{R}$ is supported on isomorphisms if $\mathscr{X}(f)$ is the zero map if $f$ is not an isomorphism.

If $\mathscr{X}$ is supported on isomorphisms it only "sees" a disjoint collection of groupoids rather than the entire category $\mathscr{A}$.

Let $B(\mathscr{A})$ be a choice of representative for each isomorphism class of objects in $\mathscr{A}$. 
Lemma 3.6 If $\mathscr{X}: \mathscr{A l}^{\mathrm{op}} \rightarrow \mathrm{Ch}_{R}$ and $\mathscr{Y}: \mathscr{A} \rightarrow \mathrm{Ch}_{R}$ are supported on isomorphisms then

$$
\mathscr{X} \odot \mathscr{Y} \cong \bigoplus_{c \in B(\mathscr{A})} \mathscr{X}(c) \otimes_{\mathscr{A}(c, c)} \mathscr{Y}(c) .
$$

The proof of this lemma is in Section 9. The idea of the proof is to use Definition 3.5 to show that

$$
\bigoplus_{c \in B(\mathscr{A})} \mathscr{X}(c) \otimes_{\mathscr{A}(c, c)} \mathscr{Y}(c)
$$

satisfies the universal property that defines $\mathscr{X} \odot \mathscr{Y}$.

Lemma 3.7 Suppose $\mathscr{X}$ and $\mathscr{Y}$ satisfy the conditions of Lemma 3.6. If $\mathscr{X}(c)$ is dualizable as an $\mathscr{A}(c, c)$-module with dual $\mathscr{Y}(c)$ for each $c \in B(\mathscr{A})$ then $\mathscr{X}$ is dualizable with dual y.

The idea of this proof is to use Lemma 3.6 and the coevaluation and evaluation maps for each $\mathscr{L}(c)$ to define coevaluation and evaluation maps for $\mathscr{X}$. This proof can also be found in Section 9.

Another choice for $\mathscr{V}$ is the symmetric monoidal category of pointed topological spaces, $\mathrm{Top}_{*}$. The bicategory $\mathscr{E}^{\mathscr{E}} \mathrm{Top}_{*}$ has 0 -cells categories enriched in based spaces and 1 -cells distributors enriched in based spaces. The 2 -cells in $\mathscr{E}_{\text {Top }}$ are natural transformations enriched in $\mathrm{Top}_{*}$.

If $\mathscr{L}^{\mathrm{op}}: \mathscr{A} \rightarrow \mathrm{Top}_{*}$ and $\mathscr{Y}: \mathscr{A} \rightarrow \mathrm{Top}_{*}$ are enriched functors $\mathscr{X} \odot \mathscr{Y}$ is the bar resolution $B(\mathscr{X}, \mathscr{A}, \mathscr{Y})$. This is the geometric realization of the simplicial space with $n$-simplices

$$
\coprod_{a_{0}, a_{1}, \ldots, a_{n} \in \mathrm{ob} \mathscr{A}} \mathscr{X}\left(a_{0}\right) \wedge \mathscr{A}\left(a_{1}, a_{0}\right) \wedge \ldots \wedge \mathscr{A}\left(a_{n}, a_{n-1}\right) \wedge \mathscr{Y}\left(a_{n}\right) .
$$

The definition of the shadow is similar. If $\mathscr{L}: \mathscr{A} \otimes \mathscr{A}^{\mathrm{op}} \rightarrow \mathrm{Top}_{*}$ is a enriched functor, the shadow of $\mathscr{L},\langle\langle\mathscr{E}\rangle\rangle$, is the cyclic bar resolution $C(\mathscr{A}, \mathscr{L})$. This is the geometric realization of the simplicial space with $n$-simplices

$$
\coprod_{a_{0}, a_{1}, \ldots, a_{n} \in \mathrm{ob} \mathscr{A}} \mathscr{L}\left(a_{n}, a_{0}\right) \wedge \mathscr{A}\left(a_{1}, a_{0}\right) \wedge \ldots \wedge \mathscr{A}\left(a_{n}, a_{n-1}\right) .
$$

Let $\mathscr{A}$ be a category enriched in based spaces. Let $U_{\mathscr{A}}: \mathscr{A} \otimes \mathscr{A}^{\mathrm{op}} \rightarrow \mathrm{Top}_{*}$ be defined by $U_{\mathscr{A}}\left(a, a^{\prime}\right)=\mathscr{A}\left(a^{\prime}, a\right)$. Composition in $\mathscr{A}$ defines the action of $\mathscr{A}$ and $\mathscr{A}^{\text {op }}$ on $U_{\mathscr{A}}$.

Definition 3.8 An enriched functor $\mathscr{L}: \mathscr{A} \rightarrow \mathrm{Top}_{*}$ is $n$-dualizable if there is a functor $\mathscr{Y}: \mathscr{A}^{\text {op }} \rightarrow$ Top $_{*}$, a map $\eta: S^{n} \rightarrow B(\mathscr{X}, \mathscr{A}, \mathscr{Y})$, and an $\mathscr{A}-\mathscr{A}$-equivariant map $\epsilon: \mathscr{Y} \wedge \mathscr{X} \rightarrow S^{n} \wedge U_{\mathscr{A}}$ such that the usual diagrams commute up to $\mathscr{A}$-equivariant homotopy. 
We will use the ideas of Lemma 3.7 to produced dual pairs in this bicategory, but we will not prove a general characterization.

Definition 3.9 If $\mathscr{L}: \mathscr{A} \rightarrow \mathrm{Top}_{*}$ is dualizable, $\mathscr{P}: \mathscr{A} \otimes \mathscr{A}^{\mathrm{op}} \rightarrow \mathrm{Top}_{*}$ is an enriched functor and $f: \mathscr{X} \rightarrow \mathscr{X} \odot \mathscr{P}$ is an enriched natural transformation, the trace of $f$ is the stable homotopy class of the composite

$$
S^{n} \stackrel{\eta}{\longrightarrow}\langle\langle\mathscr{X} \odot \mathscr{Y}\rangle\rangle \stackrel{f \odot \text { id }}{\longrightarrow}\langle\langle\mathscr{X} \odot \mathscr{P} \odot \mathscr{Y}\rangle\rangle \stackrel{\theta}{\longrightarrow}\left\langle\langle\mathscr{P} \odot \mathscr{Y} \odot \mathscr{X}\rangle \stackrel{\text { id } \odot \epsilon}{\longrightarrow} S^{n} \wedge\langle\langle\mathscr{P}\rangle\rangle .\right.
$$

\section{The relative Lefschetz number and index}

The relative Lefschetz number and relative index can both be described using a classical approach, but we will describe them using duality and trace in a bicategory. The formal structure gives a different perspective on these invariants and is a starting point for the more complicated invariants we will consider in the later sections.

Let $A \subset B$ be topological spaces.

Definition 4.1 The relative component category $\Pi_{0}(B, A)$ of a pair $(B, A)$ has objects the points of $B$. The morphisms of $\Pi_{0}(B, A)$ are

$$
\Pi_{0}(B, A)(x, y)= \begin{cases}* & \text { if } x \in B \backslash A \text { and }[x]=[y] \in \pi_{0}(B), \\ \varnothing & \text { if } x \in B \backslash A \text { and }[x] \neq[y] \in \pi_{0}(B), \\ * & \text { if } x, y \in A \text { and }[x]=[y] \in \pi_{0}(A), \\ \varnothing & \text { if } x, y \in A \text { and }[x] \neq[y] \in \pi_{0}(A), \\ \varnothing & \text { if } x \in A, y \notin A .\end{cases}
$$

The composition is defined by the rules

$$
\begin{array}{ll}
* \circ *=* & \varnothing \circ \varnothing=\varnothing \\
\varnothing \circ *=\varnothing & * \circ \varnothing=\varnothing .
\end{array}
$$

For most pairs of spaces $A \subset B$ this category is an EI-category but not a groupoid. For example, if $x \in B \backslash A, y \in A$, and $[x]=[y] \in \pi_{0}(B)$ then $\Pi_{0}(B, A)(x, y)=*$ and $\Pi_{0}(B, A)(y, x)=\varnothing$. The relative component category is similar to the equivariant component category. See tom Dieck [4, I.10.3]. When we need to think of this category as enriched in based spaces we will add disjoint base points.

If $A$ and $B$ are connected and $B \backslash A$ is nonempty this category has two isomorphism classes of objects. 
If $x \in A$, let $A(x)$ be the component of $A$ that contains $x$. If $y \in B$, let $B(y)$ be the component of $B$ that contains $y$.

Definition 4.2 The relative component space, $\overline{B \mid A}$, of the pair $(B, A)$ is the functor

$$
\Pi_{0}(B, A)^{\mathrm{op}} \rightarrow \mathrm{Top}_{*}
$$

defined by $\quad \overline{B \mid A}(x)= \begin{cases}A(x)_{+} & \text {if } x \in A \\ B(x) \cup C(A \cap B(x)) & \text { if } x \notin A\end{cases}$

The morphisms $A(x) \rightarrow A(x)$ and $B(x) \cup C(A \cap B(x)) \rightarrow B(x) \cup C(A \cap B(x))$ are the identity maps. The map $A(x) \rightarrow B(x) \cup C(A \cap B(x))$ is the inclusion of $A(x)$ as the base point.

Recall that $C(A \cap B(x))$ is the cone on $A \cap B(x)$. The base point is the cone point. Since $A \subset B$ is assumed to be a cofibration $B(x) \cup C(A \cap B(x))$ is homotopy equivalent to $B(x) /(A \cap B(x))$.

Lemma 4.3 If $A$ and $B$ are both compact ENR's or closed smooth manifolds then $\overline{B \mid A}$ is dualizable.

Remark 4.4 Starting with the proof of this theorem we will focus on the case of closed smooth manifolds. The results in this section and Section 5 and Section 6 have versions for compact ENR's as well. The statements and proofs for compact ENR's are very similar to those for closed smooth manifolds.

Some of the results in Section 7 have only been shown for manifolds.

Proof of Lemma 4.3 Define a functor $D(\overline{B \mid A}): \Pi_{0}(B, A) \rightarrow$ Top $_{*}$ by

$$
D(\overline{B \mid A})(x)= \begin{cases}D\left(A(x)_{+}\right) & \text {if } x \in A, \\ D(B(x) \cup C(A \cap B(x))) & \text { if } x \notin A\end{cases}
$$

where $D\left(A(x)_{+}\right)$and $D(B(x) \cup C(A \cap B(x)))$ denote the duals of $A(x)_{+}$and $B(x) \cup C(A \cap B(x))$ as described in Proposition 1.5. The morphisms are the identity maps or the inclusion.

To simplify notation, consider the case where $A$ and $B$ are both connected and $A \neq B$. The general case is similar. The evaluation for this dual pair is a natural transformation

$$
\epsilon: D(\overline{B \mid A}) \odot \overline{B \mid A} \rightarrow S^{n} \wedge\left(\Pi_{0}(B, A)\right)_{+} .
$$


Let $x \in A$ and $y \in B \backslash A$ represent the isomorphism classes of objects of $\Pi_{0}(B, A)$. Then $\epsilon$ consists of four maps:

$$
\begin{gathered}
D(B \cup C A) \wedge(B \cup C A) \rightarrow S^{n} \\
D\left(A_{+}\right) \wedge(B \cup C A) \rightarrow S^{n} \\
D(B \cup C A) \wedge A_{+} \rightarrow * \\
D\left(A_{+}\right) \wedge A_{+} \rightarrow S^{n}
\end{gathered}
$$

By naturality, the second map must be the constant map to a point. Since $A_{+}$and $B \cup C A$ are both dualizable, the evaluations for these dual pairs are the first and fourth maps.

Note that $B\left(\overline{B \mid A}, \Pi_{0}(B, A), D(\overline{B \mid A})\right)$ is equivalent to

$$
\left(A_{+} \wedge D\left(A_{+}\right)\right) \vee[(B \cup C A) \wedge D(B \cup C A)] .
$$

The dualizability of $A_{+}$and $B \cup C A$ provide coevaluation maps

$$
\begin{gathered}
\eta_{A}: S^{n} \rightarrow A_{+} \wedge D\left(A_{+}\right) \\
\eta_{B \cup C A}: S^{n} \rightarrow B \cup C A \wedge D(B \cup C A) .
\end{gathered}
$$

The coevaluation for this dual pair is the composite

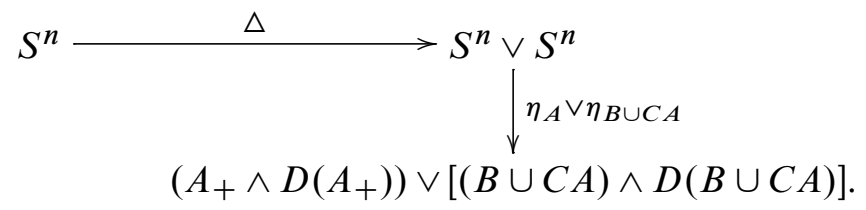

Verifying that these maps describe a dual pair can be checked for $A_{+}$and $B \cup C A$ separately. The conditions reduce to conditions checked for Proposition 1.5.

Let $\Pi_{0}^{f}(B, A)$ be the functor

$$
\Pi_{0}(B, A) \times \Pi_{0}(B, A)^{\mathrm{op}} \rightarrow \mathrm{Top}_{*}
$$

defined by $\Pi_{0}^{f}(B, A)(x, y)=\Pi_{0}(B, A)(f(y), x)_{+}$. The left action is composition. The right action is given by applying $f$ and then the composition.

A relative map $f:(B, A) \rightarrow(B, A)$ induces a natural transformation

$$
\bar{f}: \overline{B \mid A} \rightarrow \overline{B \mid A} \odot \Pi_{0}^{f}(B, A) .
$$

Since $\overline{B \mid A}$ is dualizable, the trace of $\bar{f}$ is defined.

Definition 4.5 The relative index of $f, i_{B \mid A}(f)$, is the trace of $\bar{f}$. 
The relative index is the stable homotopy class of a map

$$
S^{0} \rightarrow\left\langle\left\langle\Pi_{0}^{f}(B, A)\right\rangle\right\rangle_{+}
$$

and so it is an element of the $0-$ th stable homotopy group of $\left\langle\left\langle\Pi_{0}^{f}(B, A)\right\rangle\right\rangle_{+}$. This group is denoted $\pi_{0}^{s}\left(\left\langle\left\langle\Pi_{0}^{f}(B, A)\right\rangle\right\rangle_{+}\right)$. It is the free abelian group on the set $\left\langle\left\langle\Pi_{0}^{f}(B, A)\right\rangle\right\rangle$. Since the relative index is defined to be the trace of $\bar{f}$ it is an invariant of the relative homotopy class of $f$.

Let $\left\langle\left\langle\Pi_{0}^{f}(A)\right\rangle\right\rangle$ be

$$
\left\{[x] \in \pi_{0}(A) \mid\left[\left.f\right|_{A}(x)\right]=[x]\right\}
$$

and similarly for $B$.

Lemma 4.6 There is an isomorphism

$$
\left\langle\left\langle\Pi_{0}^{f}(B, A)\right\rangle\right\rangle \cong\left\langle\left\langle\Pi_{0}^{f}(A)\right\rangle\right\rangle \amalg\left\langle\left\langle\Pi_{0}^{f}(B)\right\rangle\right\rangle
$$

and the image of $i_{B \mid A}(f)$ under this map is

$$
\sum_{x \in\left\langle\left\langle\Pi_{0}^{f}(A)\right\rangle\right\rangle} i(f, \operatorname{Fix}(f) \cap A(x))[x]+\sum_{y \in\left\langle\left\langle\Pi_{0}^{f}(B)\right\rangle\right\rangle} i(f, \operatorname{Fix}(f) \cap(B(y) \backslash A))[y] .
$$

Since $f$ is taut $i(f, \operatorname{Fix}(f) \cap A)=i\left(\left.f\right|_{A}, \operatorname{Fix}(f) \cap A\right)$ and

$$
i(f, \operatorname{Fix}(f) \cap(B \backslash A))=i(f, \operatorname{Fix}(f))-i\left(\left.f\right|_{A}, \operatorname{Fix}(f) \cap A\right) .
$$

The first equality follows from commutativity of the index and the definition of a taut map. See Zhao [31, Proposition 3.5] for the second equality.

Proof Assume $A$ and $B$ are connected and $A$ is a proper subset of $B$. These assumptions restrict the number of components of $\left\langle\left\langle\Pi_{0}^{f}(B, A)\right\rangle\right\rangle$. The proof is similar for the general case.

The set $\left\langle\left\langle\Pi_{0}^{f}(B, A)\right\rangle\right\rangle$ is defined to be the coequalizer

$$
\bigsqcup_{x, y} \Pi_{0}(B, A)(x, y) \times \Pi_{0}^{f}(B, A)(y, x) \rightrightarrows \bigsqcup_{x} \Pi_{0}^{f}(B, A)(x, x) \rightarrow\left\langle\left\langle\Pi_{0}^{f}(B, A)\right\rangle .\right.
$$

Since $\Pi_{0}(B, A)(x, y)$ is empty if $x \in A$ and $y \notin A$, this coequalizer splits as two coequalizers. One is over pairs $(x, y)$ where $x, y \in A$ and the other is over pairs $(x, y)$ where $x, y \notin A$. Each of these coequalizers consists of a single element.

This injection is compatible with Lemma 4.3 so the image of $i_{B \mid A}(f)$ under the projection to the first summand is the trace of $f$ restricted to $A$. As observed after Proposition 1.5 this is the fixed point index of $\left.f\right|_{A}$. 
The image of $i_{B \mid A}(f)$ under the projection to the second summand is the trace of $f / A: B / A \rightarrow B / A$. The fixed points of $f / A$ are the fixed points of $\left.f\right|_{B \backslash A}$ and the point that represents $A$. The point that represents $A$ is the base point and so its index does not contribute to the trace of $f / A$; see Lewis, May and Steinberger [16, Remark III.8.5].

The second component of $i_{B \mid A}(f)$ is the index defined by Jezierski [11, Theorem 1.1].

Example 4.7 Let $J$ be a nonempty, proper, connected subinterval of $S^{1}$. Let $f:\left(S^{1}, J\right) \rightarrow\left(S^{1}, J\right)$ be the identity map. Then $i_{S^{1} \mid J}(f)=(1,-1)$.

Corollary 4.8 If $f:(B, A) \rightarrow(B, A)$ has no fixed points then $i_{B \mid A}(f)=0$.

Proof Since $f$ has no fixed points $i(f)=0$. To compute the relative index of $f$ we replace $f$ by a relatively homotopic map $g$ that is taut. The map $g$ can be chosen so that $\left.f\right|_{A}=\left.g\right|_{A}$. Then $i(g)=0$ and $i\left(\left.g\right|_{A}, \operatorname{Fix}(g) \cap A\right)=i\left(\left.f\right|_{A}, \operatorname{Fix}(f) \cap A\right)=0$.

Since $f$ has no fixed points and $\left.f\right|_{A}=\left.g\right|_{A}, i(g, \operatorname{Fix}(g) \cap A)=0$. Since $g$ is taut,

$$
i(g, \operatorname{Fix}(g) \cap(B \backslash A))=i(g, \operatorname{Fix}(g))-i\left(\left.g\right|_{A}, \operatorname{Fix}(g) \cap A\right)=0 .
$$

Let $\mathbb{Q} \Pi_{0}(B, A)$ be the category with the same objects as $\Pi_{0}(B, A)$. For objects $x$ and $y$ of $\Pi_{0}(B, A)$

$$
\mathbb{Q} \Pi_{0}(B, A)(x, y)
$$

is the rational vector space on $\Pi_{0}(B, A)(x, y)$. Composing $\overline{B \mid A}$ with the rational homology functor defines a functor

$$
H_{*}(\overline{B \mid A}): \mathbb{Q} \Pi_{0}(B, A) \rightarrow \mathrm{Ch}_{\mathbb{Q}} .
$$

Proposition 4.9 If $A \subset B$ are closed smooth manifolds, then $H_{*}(\overline{B \mid A})$ is dualizable.

Proof There are two ways to prove this proposition. First, the reduced rational homology functor is strong symmetric monoidal, so this follows from Proposition 2.5.

We can also show $H_{*}(\overline{B \mid A})$ is dualizable directly by describing the coevaluation and evaluation. The functor $H_{*}(\overline{B \mid A})$ is supported on isomorphisms and so it is enough to construct a coevaluation and evaluation for the chain complexes of vector spaces $H_{*}(A)$ and $H_{*}(B, A)$. These are both finite dimensional, and so they are both dualizable with duals as in Section 1. 
A relative map $f:(B, A) \rightarrow(B, A)$ induces a map

$$
H_{*}(f): H_{*}(\overline{B \mid A}) \rightarrow H_{*}(\overline{B \mid A}) \odot \mathbb{Q} \Pi_{0}^{f}(B, A)
$$

by applying the rational homology functor to $\bar{f}$.

Definition 4.10 The relative Lefschetz number of $f, L_{B \mid A}(f)$, is the trace of $H_{*}(f)$.

Lemma 4.11 The image of $L_{B \mid A}(f)$ under the isomorphism in Lemma 4.6 is

$$
\sum_{x \in\left\langle\left\langle\Pi_{0}^{f}(A)\right\rangle\right.} L_{A(x)}(f)[x]+\sum_{y \in\left\langle\left\langle\Pi_{0}^{f}(B)\right\rangle\right.} L_{B(y) \cup C(A \cap B(y))}(f)[y] .
$$

Here $L_{A(x)}(f)$ and $L_{B(y) \cup C(A \cap B(y))}(f)$ are the classical Lefschetz numbers of $\left.f\right|_{A(x)_{+}}$and $\left.f\right|_{B(y) \cup C(A \cap B(y))}$.

Proof Using Proposition 4.9 this proof is similar to the proof of Lemma 4.6.

The second component of $L_{B \mid A}(f)$ in Lemma 4.11 is the relative Lefschetz number defined by Bowszyc [1, Section 3].

Proposition 4.12 In $\mathbb{Q}\left\langle\left\langle\Pi_{0}^{f}(B, A)\right\rangle\right\rangle, L_{B \mid A}(f)=i_{B \mid A}(f)$.

Proof This proposition follows from Proposition 2.5 and the observation that the rational homology functor is strong symmetric monoidal.

This proposition and Corollary 4.8 imply the relative Lefschetz fixed point theorem.

Theorem A (Relative Lefschetz fixed point theorem) Let $A \subset B$ be closed smooth manifolds and $f:(B, A) \rightarrow(B, A)$ be a relative map. If $f$ has no fixed points then $L_{B \mid A}(f)=0$.

Further, if $L_{B \mid A}(f) \neq 0$ all maps relatively homotopic to $f$ have a fixed point.

\section{The geometric Reidemeister trace}

To prove a converse to Theorem A it is necessary to introduce refinements of the invariants defined in the previous section. The first of these invariants is the geometric Reidemeister trace. This is a refinement of the relative index and it will serve as a transition between the global Reidemeister trace in Section 6 and the invariant defined in Section 7. 
As for the invariants in the previous section, it is possible to define the geometric Reidemeister trace by generalizing the standard approach of fixed point indices and fixed point classes. Also as in the previous section, we do not use that approach here. Instead we use duality and trace in bicategories with shadows. This perspective gives simple comparisons of different invariants and also unifies the descriptions of different forms of the Reidemeister trace with the Lefschetz number.

Definition 5.1 The relative fundamental category, $\Pi_{1}(B, A)$, of the pair $(B, A)$ has objects the points of $B$. The morphisms $\Pi_{1}(B, A)(x, y)$ are the homotopy classes of paths from $x$ to $y$ in $A$ if $x \in A$ and homotopy classes of paths from $x$ to $y$ in $B$ if $x \in B \backslash A$.

The relative fundamental category is a subcategory of the fundamental groupoid of $B$. In most cases it is not a groupoid. For example, if $A$ and $B$ are both path connected, $x \in A$, and $y \in B \backslash A$ then $\Pi_{1}(B, A)(x, y)$ is empty and $\Pi_{1}(B, A)(y, x)$ is nonempty. This category is an EI-category. This category is similar to the equivariant fundamental category. See tom Dieck [4, I.10.7].

For $x \in A$, let $\tilde{A}_{x}$ be the universal cover of $A$ based at $x$. We think of points in $\tilde{A}_{x}$ as homotopy classes of paths in $A$ that start at $x$. For $y \in B \backslash A$ let $\widetilde{B}_{y}$ be the universal cover of $B$ based at $y$. Let $p: \widetilde{B}_{y} \rightarrow B$ be the quotient map and $\bar{A}_{y}=p^{-1}(A) \subset \widetilde{B}_{y}$.

Definition 5.2 The relative universal cover of the pair $(B, A)$ is the functor

$\begin{aligned} \widetilde{B \mid A}: & \Pi_{1}(B, A)^{\mathrm{op}} \rightarrow \mathrm{Top}_{*} \\ \text { defined by } & \widetilde{B \mid A}(x)= \begin{cases}\left(\widetilde{A}_{x}\right)_{+} & \text {if } x \in A, \\ \widetilde{B}_{x} \cup C \bar{A}_{x} & \text { if } x \notin A\end{cases} \end{aligned}$

on objects and by composition of paths on morphisms.

Lemma 5.3 If $A \subset B$ is a cofibration $\widetilde{B}_{x} / \bar{A}_{x}$ is $\pi_{1}(B)$-homotopy equivalent to $\widetilde{B}_{x} \cup C \bar{A}_{x}$.

Proof There is a $\pi_{1}(B)$-equivariant map

$$
\phi: \widetilde{B}_{x} \cup C \bar{A}_{x} \rightarrow \widetilde{B}_{x} / \bar{A}_{x}
$$

defined by collapsing the cone to the base point.

Since $A \subset B$ is a cofibration there is a map

$$
u: B \rightarrow I
$$


such that $u^{-1}(0)=A$ and a homotopy

$$
h: B \times I \rightarrow B
$$

such that $h(b, 0)=b$ for all $b \in B, h(a, t)=a$ for all $a \in A$ and $t \in I$ and $h(b, 1) \in A$ if $u(b)<1$. The map

$$
\psi: \widetilde{B}_{x} / \bar{A}_{x} \rightarrow \widetilde{B}_{x} \cup C \bar{A}_{x}
$$

is defined by

$$
\psi(\gamma)= \begin{cases}\left.h(\gamma(1), t)\right|_{[0,2(1-u(\gamma(1)))]} \circ \gamma & \text { if } \frac{1}{2} \leq u(\gamma(1)) \leq 1, \\ (h(\gamma(1), t) \circ \gamma, 1-2 u(\gamma(1))) & \text { if } 0 \leq u(\gamma(1)) \leq \frac{1}{2}\end{cases}
$$

The map $\psi$ is $\pi_{1}(B)$-equivariant. Up to homotopy it is an inverse for $\phi$.

Theorem 5.4 If $A \subset B$ are closed smooth manifolds the relative universal cover $\widetilde{B \mid A}$ is dualizable.

Proof The proof of this lemma is very similar to the proof of Lemma 4.3. We will define this dual pair by defining a dual pair for each isomorphism class of objects in $\Pi_{1}(B, A)$.

To simplify notation, consider the case where $A$ and $B$ are connected and $A$ is a proper subset of $B$. There are two isomorphism classes of objects in $\Pi_{1}(B, A)$. Choose representatives for each of these classes.

Let $S^{\nu_{A}}$ be the fiberwise one point compactification of the normal bundle of $A$. This is a space over $A$ and has a section given by the points at infinity. Let $D\left(\tilde{A}_{+}\right)$be the space $\left(\tilde{A} \times_{A} S^{\nu_{A}}\right) / \sim$ where all points of the form $\left(\gamma, \infty_{\gamma(1)}\right)$ are identified to a single point. This is the dual of $\tilde{A}_{+}$as a distributor over $\pi_{1}(A)$; see Ponto [21, Lemma 5.3.3].

Let $C_{B}\left(S^{v_{B}}, S^{v_{A}}\right)$ be

$$
(B \times\{0\}) \cup\left(S^{\nu_{A}} \times I\right) \cup\left(S^{\nu_{B}} \times\{1\}\right) .
$$

This is the fiberwise cone of the map $S^{\nu_{A}} \rightarrow S^{\nu_{B}}$ over $B$. Let $D(\widetilde{B} \cup C \bar{A})$ be the space

$$
\left(\widetilde{B} \times_{B} C_{B}\left(S^{\nu_{B}}, S^{\nu_{A}}\right)\right) / \sim
$$

where all points of the form $(\gamma, \gamma(1) \times\{1\})$ are identified to a single point. This is the $\odot$ composition of the fiberwise spaces $(\widetilde{B}, p)_{+}$and $C_{B}\left(S^{\nu_{B}}, S^{\nu_{A}}\right)$ defined by May and Sigurdsson [18, Construction 17.1.3]. An argument like that in [21, Lemma 5.3.3] for $\widetilde{A}_{+}$shows this is the dual of $\widetilde{B} \cup C \bar{A}$ as a distributor over $\pi_{1}(B)$. 
The dual of $\widetilde{B \mid A}$, denoted $D(\widetilde{B \mid A})$, is

$$
D(\widetilde{B \mid A})(x)= \begin{cases}D\left(\widetilde{A}_{+}\right) & \text {if } x \in A, \\ D(\widetilde{B} \cup C \bar{A}) & \text { if } x \in B \backslash A .\end{cases}
$$

The action of the morphisms in $\Pi_{1}(B, A)$ is by composition.

As in Lemma 4.3, there are four maps that define the natural transformation $\epsilon$. Exactly as in that case there are only two that are nontrivial. These maps are the evaluation maps for the dual pairs $\left(\tilde{A}_{+}, D\left(\tilde{A}_{+}\right)\right)$and $(\widetilde{B} \cup C \bar{A}, D(\widetilde{B} \cup C \bar{A}))$.

Also as in Lemma 4.3, $B\left(\widetilde{B \mid A}, \Pi_{1}(B, A), D(\widetilde{B \mid A})\right)$ is equivalent to

$$
\left(\tilde{A}_{+} \wedge_{\pi_{1}(A)} D\left(\tilde{A}_{+}\right)\right) \vee\left((\widetilde{B} \cup C \bar{A}) \wedge_{\pi_{1}(B)} D(\tilde{B} \cup C \bar{A})\right) .
$$

The coevaluation map is the composite of the fold map

$$
S^{n} \rightarrow S^{n} \vee S^{n}
$$

and the coevaluations for the pairs $\left(\tilde{A}_{+}, D\left(\tilde{A}_{+}\right)\right)$and $(\widetilde{B} \cup C \bar{A}, D(\widetilde{B} \cup C \bar{A}))$.

The required diagrams commute since the coevaluation and evaluation maps are defined using coevaluation and evaluation maps from the dual pairs $\left(\tilde{A}_{+}, D\left(\widetilde{A}_{+}\right)\right)$and $(\widetilde{B} \cup C \bar{A}, D(\widetilde{B} \cup C \bar{A}))$.

Remark 5.5 Later we will need more explicit descriptions of the coevaluation and evaluation maps for the pairs $\left(\widetilde{A}_{+}, D\left(\widetilde{A}_{+}\right)\right)$and $(\widetilde{B} \cup C \bar{A}, D(\widetilde{B} \cup C \bar{A}))$.

The coevaluation for the pair $\left(\tilde{A}_{+}, D\left(\tilde{A}_{+}\right)\right)$is the composite

$$
S^{n} \longrightarrow T v_{A} \longrightarrow \tilde{A}_{+} \wedge_{\pi_{1} A} D\left(\tilde{A}_{+}\right)
$$

of the Pontryagin-Thom map for an embedding of $A$ in $\mathbb{R}^{n}$ with the map $v \mapsto(\gamma, \gamma, v)$ where $\gamma$ is any element of $\widetilde{A}$ that ends at the base of $v$.

Since $A$ is locally contractible there is a neighborhood $U$ of the diagonal in $A \times A$ and a map

$$
H: V \rightarrow A^{I}
$$

that satisfies $H(x, x)(t)=x, H(x, y, 0)=x$, and $H(x, y, 1)=y$. The evaluation for the pair $\left(\tilde{A}_{+}, D\left(\tilde{A}_{+}\right)\right)$,

$$
D\left(\widetilde{A}_{+}\right) \wedge \widetilde{A}_{+} \rightarrow S^{n} \wedge \pi_{1} A_{+}
$$

is defined by

$$
(v, \gamma, \delta)=\left(\epsilon(v, \delta(1)), \gamma^{-1} H(\delta(1), \gamma(1)) \delta\right)
$$

where $\epsilon$ is the evaluation for the dual pair $\left(A_{+}, D\left(A_{+}\right)\right)$.

The coevaluation and evaluation for the dual pair $(\widetilde{B} \cup C \bar{A}, D(\widetilde{B} \cup C \bar{A}))$ are similar. 
A relative map $f:(B, A) \rightarrow(B, A)$ induces a map

$$
f_{*}: \widetilde{B \mid A} \rightarrow \widetilde{B \mid A} \odot \Pi_{1}^{f}(B, A)
$$

where $\Pi_{1}^{f}(B, A)(x, y)=\Pi_{1}(B, A)(f(y), x)_{+}$. The left action of $\Pi_{1}(B, A)$ on $\Pi_{1}^{f}(B, A)$ is the usual left action. The right action of $\Pi_{1}(B, A)$ on $\Pi_{1}^{f}(B, A)$ is given by applying $f$ and then composition.

Definition 5.6 The relative geometric Reidemeister trace of $f:(B, A) \rightarrow(B, A)$, $R_{B \mid A}^{\mathrm{ge}}(f)$, is the trace of the map

$$
f_{*}: \widetilde{B \mid A} \rightarrow \widetilde{B \mid A} \odot \Pi_{1}^{f}(B, A) .
$$

The relative geometric Reidemeister trace is the stable homotopy class of a map

$$
S^{0} \rightarrow\left\langle\left\langle\Pi_{1}^{f}(B, A)\right\rangle_{+}\right.
$$

and so it is an element of the 0 -th stable homotopy group of $\left\langle\left\langle\Pi_{1}^{f}(B, A)\right\rangle_{+}\right.$. The relative geometric Reidemeister trace is an invariant of the relative homotopy class of the map.

Let $X$ be a dualizable space. For a space $U$ and a map

$$
\triangle: X \rightarrow X \wedge U
$$

the transfer of an endomorphism $f: X \rightarrow X$ with respect to $\triangle$ is the composite

$$
S^{n} \stackrel{\eta}{\longrightarrow} X \wedge D X \stackrel{\gamma}{\longrightarrow} D X \wedge X \stackrel{\mathrm{id} \wedge f}{\longrightarrow} D X \wedge X \stackrel{\mathrm{id} \wedge \triangle}{\longrightarrow} D X \wedge X \wedge U \stackrel{\epsilon \wedge \text { id }}{\longrightarrow} S^{n} \wedge U .
$$

Let

$$
\Lambda^{\left.f\right|_{A} A}:=\left\{\gamma \in A^{I} \mid f(\gamma(0))=\gamma(1)\right\}
$$

and

$$
\Lambda^{f} B:=\left\{\gamma \in B^{I} \mid f(\gamma(0))=\gamma(1)\right\} \text {. }
$$

Since $A$ and $B$ are locally contractible and $f$ is taut there are neighborhoods $U_{A}$ of the fixed points of $A$ and $U_{B}$ of the fixed points of $B \backslash A$ and maps

$$
\begin{gathered}
\iota_{A}: U_{A} \rightarrow \Lambda^{\left.f\right|_{A} A} \\
\iota_{B}: U_{B} \rightarrow \Lambda^{f} B
\end{gathered}
$$

that take a fixed point of $f$ to the constant path at that point.

Let $\tau_{U_{A}}\left(\left.f\right|_{A}\right)$ denote the transfer of $f$ with respect to the diagonal map

$$
A_{+} \rightarrow A_{+} \wedge \overline{U_{A}} / \partial\left(\overline{U_{A}}\right)
$$

and similarly for $B$. 
Lemma 5.7 If $A$ is a proper subset of $B$ there is an isomorphism

$$
\pi_{0}^{s}\left(\left\langle\left\langle\Pi_{1}^{f}(B, A)\right\rangle_{+}\right) \cong \pi_{0}^{s}\left(\Lambda^{\left.f\right|_{A}} A_{+}\right) \oplus \pi_{0}^{s}\left(\Lambda^{f} B_{+}\right) .\right.
$$

The image of the relative geometric Reidemeister trace of $f$ under this isomorphism is

$$
\left(\iota_{A}\right)_{*}\left(\tau_{U_{A}}\left(\left.f\right|_{A}\right)\right)+\left(\iota_{B}\right)_{*}\left(\tau_{U_{B}}(f)\right) .
$$

Proof We first define the isomorphism.

Note that $\pi_{0}^{s}\left(X_{+}\right) \cong \mathbb{Z} \pi_{0}(X)$ for any space $X$, so it is enough to show $\pi_{0}\left(\left.\Lambda^{f}\right|_{A} A\right) \oplus$ $\pi_{0}\left(\Lambda^{f} B\right)$ satisfies the universal property that defines the shadow of $\Pi_{1}^{f}(B, A)$.

The shadow of $\Pi_{1}^{f}(B, A)$ is defined to be the coequalizer of the maps

$$
\bigsqcup_{x, y} \Pi_{1}(B, A)(x, y) \times \Pi_{1}(B, A)(f(y), x) \Longrightarrow \bigsqcup_{x} \Pi_{1}(B, A)(f(x), x) .
$$

The inclusion maps

$$
\begin{aligned}
& \left(\bigsqcup_{x \in A} \Pi_{1}(B, A)(f(x), x)\right) \amalg\left(\bigsqcup_{x \notin A} \Pi_{1}(B, A)(f(x), x)\right) \\
& \rightarrow \pi_{0}\left(\left.\Lambda^{f}\right|_{A} A\right) \oplus \pi_{0}\left(\Lambda^{f} B\right)
\end{aligned}
$$

define a map

$$
\theta: \bigsqcup_{x} \Pi_{1}(B, A)(f(x), x) \rightarrow \pi_{0}\left(\Lambda^{\left.f\right|_{A}} A\right) \oplus \pi_{0}\left(\Lambda^{f} B\right) .
$$

Let $\alpha \in \Pi_{1}(B, A)(x, y)$ and $\beta \in \Pi_{1}(B, A)(f(y), x)$. If $x, y \in A$ then $\beta \alpha$ and $f(\alpha) \beta$ represent the same element in $\pi_{0}\left(\Lambda^{\left.f\right|_{A}} A\right)$. If $x, y \in B \backslash A, \beta \alpha$ and $f(\alpha) \beta$ represent the same element in $\pi_{0}\left(\Lambda^{f} B\right)$. If $x$ and $y$ do not satisfy these conditions, there is no condition to check on the paths. So $\theta$ coequalizes.

If $\phi: \coprod_{x} \Pi_{1}(B, A)(f(x), x) \rightarrow M$ is a map that coequalizes the maps above define a map

$$
\bar{\phi}: \pi_{0}\left(\Lambda^{\left.f\right|_{A}} A\right) \oplus \pi_{0}\left(\Lambda^{f} B\right) \rightarrow M
$$

by $\bar{\phi}(\gamma)=\phi(\beta)$ where $\beta$ is any element of $\Pi_{1}(B, A)(f(x), x)$ that maps to $\gamma$ in $\pi_{0}\left(\left.\Lambda^{f \mid}\right|_{A} A\right) \oplus \pi_{0}\left(\Lambda^{f} B\right)$. This is independent of choices since if $\alpha$ is another lift of $\gamma$ there are paths $\mu$ and $v$ such that $f(\mu) \nu$ is homotopic to $\beta$ and $\nu \mu$ is homotopic to $\alpha$. Then $\bar{\phi}$ is unique and $\pi_{0}\left(\left.\Lambda^{f}\right|_{A} A\right) \oplus \pi_{0}\left(\Lambda^{f} B\right)$ is the coequalizer.

To describe the image of the geometric Reidemeister trace under this isomorphism it is enough to show the trace of

$$
\widetilde{\left.f\right|_{A}}: \tilde{A} \rightarrow \tilde{A}
$$

is $\left(\iota_{A}\right)_{*}\left(\tau_{U_{A}}\left(\left.f\right|_{A}\right)\right)$ and the trace of

$$
\widetilde{f}: \widetilde{B} / \bar{A} \rightarrow \widetilde{B} / \bar{A}
$$

is $\left(\iota_{B}\right)_{*}\left(\tau_{U_{B}}(f)\right)$. We will describe the first, the second is similar. 
In Remark 5.5 we gave explicit descriptions of the coevaluation and evaluation for the dual pair $\left(\tilde{A}_{+}, D\left(\tilde{A}_{+}\right)\right)$. Let $q: D\left(\tilde{A}_{+}\right) \wedge \tilde{A}_{+} \rightarrow D A \wedge A_{+}$be the quotient map. If $\eta_{1}$ and $\epsilon_{1}$ are the coevaluation and evaluation for the dual pair $\left(\tilde{A}_{+}, D\left(\tilde{A}_{+}\right)\right)$and $\eta_{2}$ and $\epsilon_{2}$ are the coevaluation and evaluation for the dual pair $\left(A_{+}, D A_{+}\right)$the explicit descriptions of $\eta_{1}$ and $\epsilon_{1}$ show that the following two diagrams commute:

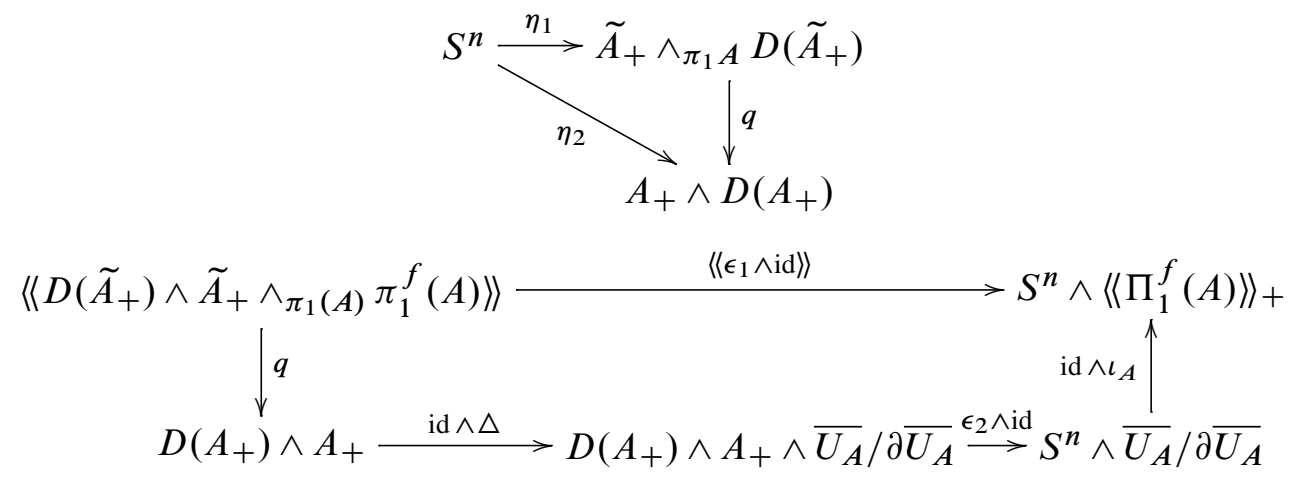

Together these diagrams show

$$
\begin{aligned}
\left.\left\langle\left\langle\epsilon_{1} \wedge \mathrm{id}\right\rangle\right\rangle\langle\tilde{f} \wedge \mathrm{id}\rangle\right\rangle \theta \eta_{1} & =\left(\mathrm{id} \wedge \iota_{A}\right)\left(\epsilon_{2} \wedge \mathrm{id}\right)(\mathrm{id} \wedge \triangle) q\left\langle\langle\tilde{f} \wedge \mathrm{id}\rangle \theta \eta_{1}\right. \\
& =\left(\mathrm{id} \wedge \iota_{A}\right)\left(\epsilon_{2} \wedge \mathrm{id}\right)(\mathrm{id} \wedge \triangle)(f \wedge \mathrm{id}) \gamma q \eta_{1} \\
& =\left(\mathrm{id} \wedge \iota_{A}\right)\left(\epsilon_{2} \wedge \mathrm{id}\right)(\mathrm{id} \wedge \triangle)(f \wedge \mathrm{id}) \gamma \eta_{2}
\end{aligned}
$$

The first composite is the trace of $\widetilde{\left.f\right|_{A}}$. The last composite is $\left(\iota_{A}\right)_{*}\left(\tau_{U_{A}}\left(\left.f\right|_{A}\right)\right)$.

For a fixed point class $\beta$ of $f: B \rightarrow B$ let $i_{\beta}^{\text {rel }}$ be the index of the fixed points associated to $\beta$ that are contained in $B \backslash A$. For a fixed point class $\alpha$ of $\left.f\right|_{A}: A \rightarrow A$, let $i_{\alpha}$ be the index of the fixed points associated to $\alpha$. Since the map $f$ is taut, $i_{\alpha}$ is the fixed point index of the fixed points in $A$ with respect to either $\left.f\right|_{A}$ or $f$.

The following corollary is a consequence of Lemma 5.7 and is the generalization of Lemma 4.6. This corollary identifies the relative geometric Reidemeister trace with the generalization of the classical description of the Reidemeister trace.

Corollary 5.8 If the fixed points of $f$ are isolated,

$$
R_{B \mid A}^{\mathrm{ge}}(f)=\left(\sum i_{\alpha} \alpha\right)+\left(\sum i_{\beta}^{\mathrm{rel}} \beta\right) \in \mathbb{Z} \pi_{0}\left(\Lambda^{\left.f\right|_{A}} A\right) \oplus \mathbb{Z} \pi_{0}\left(\Lambda^{f} B\right) .
$$

The following two examples were considered by Norton-Odenthal and Wong [19]. In that paper the generalized Lefschetz number and one form of the relative Nielsen number are computed. 
Example 5.9 [19, Example 5.1] Let $B=D^{2} \times S^{1}$ and $A=S^{1} \times S^{1}$. Let $f: B \rightarrow B$ be

$$
f\left(r e^{i \theta}, e^{i t}\right)=\left(f_{1}(r) e^{-i \theta}, e^{3 i t}\right)
$$

where $f_{1}:[0,1] \rightarrow[0,1]$ is a continuous function such that $f_{1}(0)=0, f_{1}(1)=1$ and $f_{1}$ has no other fixed points. Then $f$ is a relative map with six fixed points. There are four fixed points in $A$. These fixed points all represent different fixed point classes and all have index -1 . The two fixed points outside of $A$ represent different fixed point classes in $B$ and also have index -1 .

Since $A$ is a torus, $\pi_{1}(A)=\langle a, b \mid a b a b=1\rangle$. The relation imposed on the shadow implies $\left\langle\left\langle\pi_{1}(A)^{\phi}\right\rangle\right\rangle$ consists of 4 elements,

$$
1, a, b, a b \text {. }
$$

For $B, \pi_{1}(b)=\langle b\rangle$ and $\left\langle\left\langle\pi_{1}(B)^{\phi}\right\rangle\right\rangle$ consists of 2 elements,

$$
1, b \text {. }
$$

Then

$$
R_{B \mid A}^{\mathrm{ge}}(f)=-1\left(1_{A}+a_{A}+b_{A}+a b_{A}+1_{B}+b_{B}\right) .
$$

Example 5.10 [19, Example 5.2] Let $B=S^{1} \times S^{1}$ and $A=1 \times S^{1}$. Let $f: B \rightarrow B$ be

$$
f\left(e^{i \theta}, e^{i t}\right)=\left(e^{3 i \theta}, e^{4 i t}\right) .
$$

There are three fixed points of $f$ in $A$ and three additional fixed points of $f$ in $B \backslash A$.

The three fixed points of $f$ in $A$ represent each of the three possible fixed point classes. These fixed points all have index 1 . The three fixed points in $B$ that are not in $A$ also represent three distinct fixed point classes, but these are only three of the six possible fixed point classes. These fixed points also have index 1 .

Let $\pi_{1}(B)=\langle a, b \mid a b a b=1\rangle$. Then $\pi_{1}(A)=\langle a\rangle$. The set $\left\langle\left\langle\pi_{1}(B)^{\phi}\right\rangle\right\rangle$ consists of

$$
1, a, a^{2}, b, a b, a^{2} b .
$$

The set $\left\langle\left\langle\pi_{1}(A)^{\phi}\right\rangle\right\rangle$ consists of

$$
1, a, a^{2} \text {. }
$$

Then

$$
R_{B \mid A}^{\mathrm{ge}}(f)=1_{A}+a_{A}+a_{A}^{2}+b_{B}+(a b)_{B}+\left(a^{2} b\right)_{B} .
$$

\section{The relative Nielsen number}

One of the expectations for the Reidemeister trace is that it can detect when a map has no fixed points but it does not have to provide a lower bound for the number of fixed 
points. This is very different from the Nielsen number. The goal of the Nielsen number is to provide a lower bound.

In the classical case, the Nielsen number is the number of nonzero coefficients in the Reidemeister trace. This implies the Nielsen number is zero if and only if the Reidemeister trace is zero. For more general situations the connection between nonzero coefficients of the Reidemeister trace and the Nielsen number does not hold. It remains true that the Nielsen number is zero if and only if the Reidemeister trace is zero.

The inclusion of $A$ into $B$ induces a map $\pi_{1}(A) \rightarrow \pi_{1}(B)$ and also induces a map from the fixed point classes of $A$ to the fixed point classes of $B$. A fixed point class of $f$ or $\left.f\right|_{A}$ is essential if its coefficient in the classical Reidemeister trace is nonzero. Let

$$
N\left(f,\left.f\right|_{A}\right)
$$

be the number of essential fixed point classes of $B$ that are in the image of an essential class of $A$. Let $N(f)$ be the classical Nielsen number of $f$ and $N\left(\left.f\right|_{A}\right)$ be the classical Nielsen number of $\left.f\right|_{A}$.

We recall the definition of the relative Nielsen number. See Zhao [31].

Definition 5.11 [31, Definition 2.5] The relative Nielsen number, $N(f ; B, A)$, is

$$
N\left(\left.f\right|_{A}\right)+\left(N(f)-N\left(f,\left.f\right|_{A}\right)\right) .
$$

Lemma 5.12 The relative Nielsen number of $f$ is zero if and only if the relative geometric Reidemeister trace of $f$ is zero.

Proof If the relative geometric Reidemeister trace of $f$ is zero Corollary 5.8 implies $\left(\sum i_{\beta}^{\text {rel }} \beta\right)+\left(\sum i_{\alpha} \alpha\right)$ is zero. Since $\mathbb{Z}\left\langle\left\langle\Pi_{1}^{f}(B, A)\right\rangle\right\rangle$ is a free group generated by the $\alpha$ 's and $\beta$ 's each $i_{\beta}^{\text {rel }}$ and $i_{\alpha}$ are zero. Since the $i_{\alpha}$ 's are zero, $N\left(\left.f\right|_{A}\right)$ and $N\left(f,\left.f\right|_{A}\right)$ are zero. Since each of the $i_{\alpha}$ 's are zero $i_{\beta}=i_{\beta}^{\text {rel }}=0$ for every $\beta$. This implies $N(f)$ is also zero.

By definition $N\left(\left.f\right|_{A}\right), N(f)$, and $N\left(f,\left.f\right|_{A}\right)$ are all greater than or equal to zero and $N\left(f,\left.f\right|_{A}\right) \leq N(f)$. If the relative Nielsen number of $f$ is zero $N\left(\left.f\right|_{A}\right)=0$ and $N(f)=N\left(f,\left.f\right|_{A}\right)$. Since $N\left(\left.f\right|_{A}\right)=0, N\left(f,\left.f\right|_{A}\right)=0$ and so $N(f)=0$. Since $N\left(\left.f\right|_{A}\right)=0$ all of the $i_{\alpha}$ 's are zero and $i_{\beta}^{\text {rel }}=i_{\beta}$. Since $N(f)=0, i_{\beta}=0$ for all $\beta$.

The relative Nielsen numbers for the maps in the examples above were computed by Norton-Odenthal and Wong [19]. The relative Nielsen number for Example 5.9 is 4. This is not the number of nonzero coefficients in the relative Reidemeister trace. The relative Nielsen number for Example 5.10 is 6. This does happen to be the number of 
nonzero coefficients in the relative Reidemeister trace. These numbers coincide because $N\left(f,\left.f\right|_{A}\right)$ is zero for this example.

Other references for relative Nielsen theory include Jezierski [11], Schirmer [24; 25] and Zhao $[29 ; 30]$. These invariants are also related to the Nielsen numbers for stratified spaces defined by Jiang, Zhao and Zheng [13].

\section{The global Reidemeister trace}

In this section we define the relative global Reidemeister trace. This invariant is a generalization of the relative Lefschetz number and can be identified with the relative geometric Reidemeister trace. The relative global Reidemeister trace is a relative generalization of the global Reidemeister trace defined by Husseini [9]. It is related to the invariants defined by Norton-Odenthal and Wong [19] and Zhao [32], but it is not the same as either of these invariants.

In this section we will assume $A$ and $B$ are finite $\mathrm{CW}$-complexes. Just like Remark 4.4 this simplifies the discussion.

Let $\mathbb{Z} \Pi_{1}(B, A)$ be the category with the same objects as $\Pi_{1}(B, A)$. The morphism set

$$
\mathbb{Z} \Pi_{1}(B, A)(x, y)
$$

is the free abelian group on the set $\Pi_{1}(B, A)(x, y)$.

There is a functor

$$
C_{*}(\widetilde{B \mid A}): \mathbb{Z} \Pi_{1}(B, A)^{\mathrm{op}} \rightarrow \mathrm{Ch}_{\mathbb{Z}}
$$

defined by $C_{*}(\widetilde{B \mid A})(x)=C_{*}(\widetilde{B \mid A}(x) ; \mathbb{Z})$ where the second $C_{*}$ indicates the cellular chain complex. The action of the morphisms of $\Pi_{1}(B, A)$ is induced from the action on $\widetilde{B \mid A}$. This functor is defined in the same way the functor $H_{*}(\overline{B \mid A})$ is defined from the functor $\overline{B \mid A}$ except we replace the rational homology functor with the integral chain complex functor.

Note, unlike Section 4, in this section we will use integral chains. For these invariants we could also use rational chains. Either choice will give the same invariants. In Section 4 we used rational homology since integral homology would have made the definitions more complicated.

Proposition 6.1 If $A \subset B$ are finite $C W$-complexes, then the $\mathbb{Z} \Pi_{1}(B, A)$-module $C_{*}(\widetilde{B \mid A})$ is dualizable. 
Proof Like Proposition 4.9 there are two possible proofs of this theorem.

The rational cellular chain complex functor induces a functor on bicategories, and for $A \subset B$ closed smooth manifolds, Theorem 5.4 shows that $\widetilde{B \mid A}$ is dualizable. Proposition 2.5 then implies that $C_{*}(\widetilde{B \mid A})$ is dualizable.

There is a second approach using Lemma 3.7. If $x \in A, C_{*}(\widetilde{B \mid A})(x)=C_{*}\left(\tilde{A}_{x}\right)$ as a module over $\pi_{1}(A, x)$. This is a finitely generated free module and so is dualizable with dual

$$
\operatorname{Hom}_{\mathbb{Z} \pi_{1}(A, x)}\left(C_{*}\left(\tilde{A}_{x}\right), \mathbb{Z} \pi_{1}(A, x)\right) .
$$

If $x \in B \backslash A, C_{*}(\widetilde{B \mid A})(x)=C_{*}\left(\widetilde{B}_{x}, \bar{A}_{x}\right)$ as a module over $\pi_{1}(B, x)$. This is also a finitely generated free module and so is dualizable with dual

$$
\operatorname{Hom}_{\mathbb{Z} \pi_{1}(B, x)}\left(C_{*}\left(\widetilde{B}_{x}, \bar{A}_{x}\right), \mathbb{Z} \pi_{1}(B, x)\right) .
$$

Since $C_{*}(\widetilde{B \mid A})$ is supported on isomorphisms, Lemma 3.7 implies $C_{*}(\widetilde{B \mid A})$ is dualizable.

A map $f:(B, A) \rightarrow(B, A)$ induces a map

$$
f_{*}: C_{*}(\widetilde{B \mid A}) \rightarrow C_{*}(\widetilde{B \mid A}) \odot \mathbb{Z} \Pi_{1}^{f}(B, A) .
$$

Since $C_{*}(\widetilde{B \mid A})$ is dualizable, the trace of $f_{*}$ is defined.

Definition 6.2 The relative global Reidemeister trace $R_{B \mid A}^{\mathrm{gl}}(f)$ of

$$
f:(B, A) \rightarrow(B, A)
$$

is the trace of

$$
f_{*}: C_{*}(\widetilde{B \mid A}) \rightarrow C_{*}(\widetilde{B \mid A}) \odot \mathbb{Z} \Pi_{1}^{f}(B, A)
$$

The relative global Reidemeister trace of $f$ is a map

$$
\mathbb{Z} \rightarrow \mathbb{Z}\left\langle\left\langle\Pi_{1}^{f}(B, A)\right\rangle\right\rangle .
$$

We also have a statement similar to Lemma 5.7.

Lemma 6.3 If $A$ is a proper subset of $B$ then

$$
\left\langle\langle \Pi _ { 1 } ^ { f } ( B , A ) \rangle \cong \langle \langle \Pi _ { 1 } ^ { f } ( B ) \rangle \rangle \amalg \left\langle\left\langle\Pi_{1}^{f}(A)\right\rangle .\right.\right.
$$

The image of $R_{B \mid A}^{\mathrm{gl}}(f)$ under this isomorphism is

$$
\sum_{x \in\left\langle\left\langle\Pi_{0}^{f}(A)\right\rangle\right\rangle} R^{\mathrm{gl}}\left(\left.f\right|_{A(x)}\right)[x]+\sum_{y \in\left\langle\left\langle\Pi_{0}^{f}(B)\right\rangle\right\rangle} R^{\mathrm{gl}}\left(\left.f\right|_{(B(y), B(y) \cap A)}\right)[y] .
$$


Here $R^{\mathrm{gl}}\left(\left.f\right|_{A(x)}\right)$ denotes the usual global Reidemeister trace of $\left.f\right|_{A(x)}$ as defined by Husseini in [9]. The invariant $R^{\mathrm{gl}}\left(\left.f\right|_{(B(y), B(y) \cap A)}\right)$ is the Hattori-Stallings trace of

$$
\tilde{f}_{*}: C_{*}\left(\widetilde{B}_{y},\left(\widetilde{B}_{y} \cap \bar{A}_{y}\right)\right) \rightarrow C_{*}\left(\widetilde{B}_{y},\left(\widetilde{B}_{y} \cap \bar{A}_{y}\right)\right) \otimes \pi_{1}^{f}(B, y)
$$

as a module over $\pi_{1}(B, y)$.

Proof To simplify notation, consider the case where $A$ and $B$ are connected and $A$ is a proper subset of $B$. The proof is similar if $A$ and $B$ are not connected.

The shadow is defined to be the coequalizer of the maps

$$
\bigsqcup_{x, y} \Pi_{1}(B, A)(x, y) \times \Pi_{1}(B, A)(f(y), x) \Longrightarrow \bigsqcup_{x} \Pi_{1}(B, A)(f(x), x) .
$$

Instead of indexing these coproducts over all objects in $\Pi_{1}(B, A)$ we can index over representatives of each isomorphism class of objects in $\Pi_{1}(B, A)$. This gives four terms in the first coproduct. The two cross terms are both empty and so this coequalizer splits into the coequalizer that defines $\left\langle\left\langle\Pi_{1}^{f}(B)\right\rangle\right\rangle$ and the coequalizer that defines $\left\langle\left\langle\Pi_{1}^{f}(A)\right\rangle\right\rangle$.

For the second statement, note that this isomorphism is compatible with the description of the dual pair. Then the trace is the pair of Hattori-Stallings traces.

This description of the relative global Reidemeister trace shows that the second coordinate is the relative Reidemeister trace of Zhao [32]. This also shows this invariant is related to, but not the same as, the generalized Lefschetz number defined by NortonOdenthal and Wong [19].

Proposition 6.4 If $A \subset B$ are closed smooth manifolds and $f:(B, A) \rightarrow(B, A)$ is a relative map then

$$
R_{B \mid A}^{\mathrm{ge}}(f)=R_{B \mid A}^{\mathrm{gl}}(f) .
$$

Proof Since both $R_{B \mid A}^{\mathrm{ge}}(f)$ and $R_{B \mid A}^{\mathrm{gl}}(f)$ are defined as traces and the integral cellular chain complex functor is strong symmetric monoidal this proposition follows from Proposition 2.5.

\section{A converse to the relative Lefschetz fixed point theorem}

There are several proofs of the converse to the relative Lefschetz fixed point theorem. Some, like Jezierski [11], Schirmer [24; 25] and Zhao [30], are generalizations of the simplicial arguments used in the standard proof of the converse to the classical Lefschetz fixed point theorem; see Brown [2]. 
In this section and the next we will describe a proof of the converse to the relative Lefschetz fixed point theorem that follows the outline of Klein and Williams [14; 15]. This approach is not simplicial and it easily generalizes. For example, see [15] for the equivariant generalization and Ponto [21] for the fiberwise generalization.

The approach of [14] is based on invariants that detect sections of fibrations. In the next section we prove relative generalizations of the results in [14]. In this section we apply those results to relative fixed point invariants.

The main result of this section is:

Theorem B (Converse to the relative Lefschetz fixed point theorem) Suppose $A \subset B$ are closed smooth manifolds of dimension at least 3 and the codimension of $A$ in $B$ is at least 2 . The relative global Reidemeister trace of a map

$$
f:(B, A) \rightarrow(B, A)
$$

is zero if and only if $f$ is relatively homotopic to a map with no fixed points.

The first step in the proof of Theorem B is to describe relative maps with no fixed points in terms of relative sections.

Lemma 7.1 Let $A \subset B$ be closed smooth manifolds. Relative homotopies of a map $f:(B, A) \rightarrow(B, A)$ to a relative map with no fixed points correspond to liftings in the diagram below that commute up to relative homotopy.

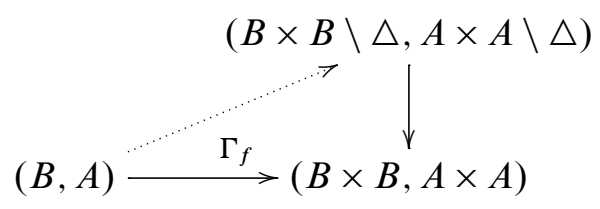

The function $\Gamma_{f}$ is the graph of $f$ and $\Gamma_{f}(m)=(m, f(m))$.

Proof If $f$ is relatively homotopic to a fixed point free map $g$ via a relative homotopy $H$, then $\Gamma_{H}$ is a relative homotopy from $\Gamma_{f}$ to $\Gamma_{g}$.

For the converse, suppose there is a relative map

$$
k:(B, A) \rightarrow(B \times B \backslash \triangle, A \times A \backslash \triangle)
$$

and a relative homotopy $K$ from $k$ to $\Gamma_{f}$.

If $A$ is a smooth manifold the first coordinate projection

$$
\operatorname{proj}_{1}: A \times A \backslash \triangle \rightarrow A
$$


is a fiber bundle and there is a lift $J_{A}$ in the diagram

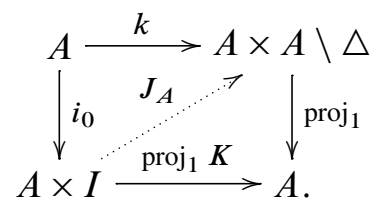

Since $A \subset B$ is a cofibration and $\operatorname{proj}_{1}: B \times B \backslash \triangle \rightarrow B$ is a fibration the diagram

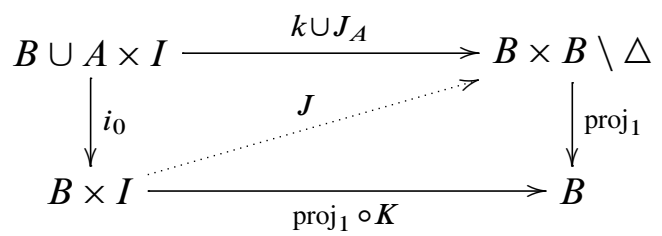

has a lift $J$ extending the lift $J_{A}$ above; see Strom [27, Theorem 4]. Note that $\operatorname{proj}_{1} \circ J(-, 1)=$ id. Let $g=\operatorname{proj}_{2} J(-, 1)$. This map has no fixed points.

The homotopies $K$ and $J$ define a relative homotopy from $\Gamma_{f}$ to $\Gamma_{g}$.

Given a map $f: V \rightarrow Y$, let $r(f): N(f) \rightarrow Y$ denote a Hurewicz fibration such that

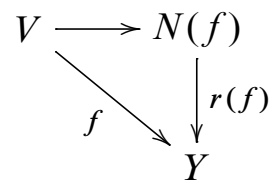

commutes and $V \rightarrow N(f)$ is an equivalence.

Lemma 7.2 Let $X \subset Y, p: M_{Y} \rightarrow Y$ be a space over $Y$ and $M_{X} \subset p^{-1}(X)$.

Liftings up to relative homotopy in the diagram

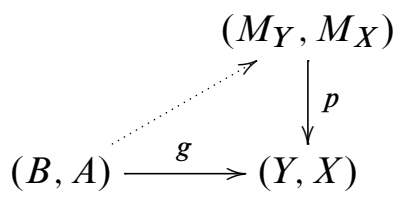

correspond to relative sections of the pair of fibrations

$$
\left(g^{*} N\left(p_{Y}\right), g^{*} N\left(p_{X}\right)\right) \rightarrow(B, A) .
$$


If $p: E \rightarrow B$ is a Hurewicz fibration the unreduced fiberwise suspension of $p$ is the double mapping cylinder

$$
S_{B} E:=B \times\{0\} \cup_{p} E \times[0,1] \cup_{p} B \times\{1\} .
$$

The map $p: E \rightarrow B$ defines a fibration

$$
p: S_{B} E \rightarrow B
$$

There are two sections of this fibration, $\sigma_{1}, \sigma_{2}: B \rightarrow S_{B} E$, defined by the inclusions of $B \times\{0\}$ and $B \times\{1\}$.

It simplifies notation to think of these sections as defining a map of ex-spaces. An ex-space $Y$ over $B$ is a space $Y$ and two continuous maps

$$
B \stackrel{s}{\rightarrow} Y \stackrel{p}{\rightarrow} B
$$

such that $p s=\operatorname{id}_{B}$. Let $S_{B}^{0}$ be the ex-space

$$
B \rightarrow B \amalg B \rightarrow B
$$

where the first map is the inclusion on the first factor and the second map is the identity on each component. The sections $\sigma_{1}$ and $\sigma_{2}$ define a fiberwise map

$$
S_{B}^{0} \rightarrow S_{B} E \text {. }
$$

For ex-spaces $X$ and $Y$ over $B$, let $[X, Y]_{B}$ be sectioned fiberwise homotopy classes of maps from $X$ to $Y$ and let $\{X, Y\}_{B}$ be stable fiberwise homotopy classes of maps from $X$ to $Y$. See May and Sigurdsson [18] for more information about the homotopy theory of ex-spaces. Using $\sigma_{1}, S_{B} E$ is an ex-space and the fiberwise map $S_{B}^{0} \rightarrow S_{B} E$ determines an element of

$$
\left[S_{B}^{0}, S_{B} E\right]_{B} .
$$

Let $i_{B}: B \times B \backslash \triangle \rightarrow B \times B$ be the inclusion. Then the pair of fibrations

$$
\left(\Gamma_{f *}\left(N\left(i_{B}\right)\right), \Gamma_{\left.f\right|_{A} *}\left(N\left(i_{A}\right)\right)\right) \rightarrow(B, A)
$$

determines an element in

$$
\left[S_{B}^{0}, S_{B} \Gamma_{f *}\left(N\left(i_{B}\right)\right)\right]_{B} \oplus\left[S_{A}^{0}, S_{A} \Gamma_{\left.f\right|_{A} *}\left(N\left(i_{A}\right)\right)\right]_{A} .
$$

This element will be denoted $R_{B \mid A}^{\mathrm{KW}}(f)$.

Proposition 7.3 Let $A \subset B$ be closed smooth manifolds of dimension at least 3 such that the codimension of $A$ in $B$ is at least 2. A continuous map

$$
f:(B, A) \rightarrow(B, A)
$$

is relatively homotopic to a map with no fixed points if and only if $R_{B \mid A}^{\mathrm{KW}}(f)=0$. 
The proof of this proposition, except for one key step proved in the next section, follows the preliminary lemma below.

Lemma 7.4 [14, Lemma 6.1, Corollary 6.2] Let $M$ be a manifold of dimension $n$, $i: M \times M \backslash \triangle \rightarrow M \times M$ be the inclusion, and $f: M \rightarrow M$ be a continuous map. Then $\Gamma_{f *}(N(i)) \rightarrow M$ is $(n-1)$-connected.

Proof of Proposition 7.3 Lemma 7.1 and Lemma 7.2 convert the question of finding a lift of a relative map $f:(B, A) \rightarrow(B, A)$ to the question of finding a section of the fibration

$$
\left(\Gamma_{f *}\left(N\left(i_{B}\right)\right),\left(\Gamma_{\left.f\right|_{A} *}\left(N\left(i_{A}\right)\right)\right) \rightarrow(B, A) .\right.
$$

If the dimension of $A$ is $n_{A}$ and the dimension of $B$ is $n_{B}$ then Lemma 7.4 implies that $\Gamma_{f *}\left(N\left(i_{B}\right)\right) \rightarrow B$ is $\left(n_{B}-1\right)$-connected and $\Gamma_{\left.f\right|_{A}}\left(N\left(i_{A}\right)\right) \rightarrow A$ is $\left(n_{A}-1\right)-$ connected. If $n_{A}$ and $n_{B}$ are at least 3 and $n_{B}-n_{A}$ is at least 2, Proposition 8.6 implies that

$$
\left(\Gamma_{f *}\left(N\left(i_{B}\right)\right), \Gamma_{\left.f\right|_{A} *}\left(N\left(i_{A}\right)\right)\right) \rightarrow(B, A)
$$

has a relative section if and only if $R_{B \mid A}^{\mathrm{KW}}(f)=0$.

The hypotheses in this proposition are not the standard hypotheses used in the converse to the relative Lefschetz fixed point theorem. The standard condition is that

$$
\pi_{1}(B \backslash A) \rightarrow \pi_{1}(B)
$$

is surjective. The codimension condition implies this condition. We use a codimension condition in Proposition 7.3 since it is compatible with the techniques used in the proof of Proposition 8.6. I don't know if the surjectivity condition can be used in this approach.

To complete the proof of Theorem B we need to compare $R_{B \mid A}^{\mathrm{KW}}(f)$ and the relative geometric Reidemeister trace.

Proposition 7.5 Let $A \subset B$ be closed smooth manifolds and $f:(B, A) \rightarrow(B, A)$ be a relative map. Then

$$
R_{B \mid A}^{\mathrm{KW}}(f)=0 \text { if and only if } R_{B \mid A}^{\mathrm{ge}}(f)=0 .
$$

We recall a lemma from [15].

Lemma 7.6 [15, Lemma 7.1; 21, Lemma 8.3.1] Let $M$ be a closed smooth manifold with normal bundle $v_{M}$. There is a weak equivalence

$$
S^{v_{M}} \odot \Gamma_{f *} S_{M \times M} N\left(i_{M}\right) \rightarrow S^{n} \wedge\left(\Lambda^{f} M_{+}\right) .
$$


Proof of Proposition 7.5 If $A$ and $B$ are both closed smooth manifolds of dimension at least three, then the dimension assumption, Lemma 7.4, and the fiberwise Freudenthal suspension theorem in James [10, Proposition 4.2] imply the maps

$$
\begin{gathered}
{\left[S_{A}^{0}, S_{A} \Gamma_{\left.f\right|_{A} *}\left(N\left(i_{A}\right)\right)\right]_{A} \rightarrow\left\{S_{A}^{0}, S_{A} \Gamma_{f *}\left(N\left(i_{A}\right)\right)\right\}_{A}} \\
{\left[S_{B}^{0}, S_{B} \Gamma_{f *}\left(N\left(i_{B}\right)\right)\right]_{B} \rightarrow\left\{S_{B}^{0}, S_{B} \Gamma_{f *}\left(N\left(i_{B}\right)\right)\right\}_{B}}
\end{gathered}
$$

are isomorphisms. Costenoble-Waner duality in May and Sigurdsson [18, Theorem 18.5.5, Construction 18.6.3] and Lemma 7.6 imply there are isomorphisms

$$
\begin{aligned}
\left\{S_{A}^{0}, S_{A} \Gamma_{\left.f\right|_{A} *}\left(N\left(i_{A}\right)\right)\right\}_{A} & \cong\left\{S^{n}, S^{\nu_{A}} \odot S_{A} \Gamma_{f *}\left(N\left(i_{A}\right)\right)\right\} \\
& \cong\left\{S^{n}, S^{n} \wedge \Lambda^{\left.f\right|_{A}} A_{+}\right\} .
\end{aligned}
$$

and

$$
\begin{aligned}
\left\{S_{B}^{0}, S_{B} \Gamma_{f *}\left(N\left(i_{B}\right)\right)\right\}_{B} & \cong\left\{S^{n}, S^{\nu_{B}} \odot S_{B} \Gamma_{f *}\left(N\left(i_{B}\right)\right)\right\} \\
& \cong\left\{S^{n}, S^{n} \wedge \Lambda^{f} B_{+}\right\} .
\end{aligned}
$$

Let $U_{A}$ be a neighborhood of the fixed points of $\left.f\right|_{A}$ such that there is a map

$$
\iota_{A}: U_{A} \rightarrow \Lambda^{\left.f\right|_{A} A}
$$

that takes a fixed point to the constant path at that point. In [21, Theorem 6.3.2] it is shown that the image of $R_{B \mid A}^{\mathrm{KW}}(f)$ in $\pi_{0}^{s}\left(\Lambda^{\left.f\right|_{A}} A_{+}\right)$is $\iota_{A}\left(\tau\left(\left.f\right|_{U_{A}}\right)\right)$.

Let $U_{B}$ be a neighborhood of the fixed points of $f$ in $B \backslash A$ such that there is a map

$$
\iota_{B}: U_{B} \rightarrow \Lambda^{f} B
$$

which takes a fixed point to the constant path at that point.

The image of $R_{B \mid A}^{\mathrm{KW}}(f)$ in $\pi_{0}^{s}\left(\Lambda^{f} B_{+}\right)$is the composite of the transfer of $f$ with respect to the diagonal map

$$
B_{+} \rightarrow B_{+} \wedge \overline{\left(U_{B} \amalg U_{A}\right)} / \partial \overline{\left(U_{B} \amalg U_{A}\right)}
$$

with the map

$$
\iota:=\iota_{A} \amalg \iota_{B}: U_{A} \amalg U_{B} \rightarrow \Lambda^{f} B .
$$

Since the transfer is additive, Dold [5, 3.17], the image of $R_{B \mid A}^{\mathrm{KW}}(f)$ in $\pi_{0}^{s}\left(\Lambda^{f} B_{+}\right)$is

$$
\iota\left(\tau_{U_{B}} \amalg U_{A}(f)\right)=\iota\left(\tau_{U_{B}}(f)+\tau_{U_{A}}\left(\left.f\right|_{A}\right)\right)=\iota\left(\tau_{U_{B}}(f)\right)+\iota\left(\tau_{U_{A}}\left(\left.f\right|_{A}\right)\right) .
$$

Then $R_{B \mid A}^{\mathrm{KW}}(f)$ is zero if and only if $\iota_{A}\left(\tau\left(\left.f\right|_{U_{A}}\right)\right)$ and $\iota\left(\tau_{U_{B}}(f)\right)+\iota\left(\tau_{U_{A}}\left(\left.f\right|_{A}\right)\right)$ are both zero. Using Lemma 5.7 these elements are zero if and only if $R_{B \mid A}^{\mathrm{ge}}(f)$ is zero.

Proof of Theorem B Proposition 7.3 implies $f$ is relatively homotopic to a fixed point free map if and only if $R_{B \mid A}^{\mathrm{KW}}(f)=0$. Proposition 7.5 implies $R_{B \mid A}^{\mathrm{KW}}(f)=0$ if and only if $R_{B \mid A}^{\mathrm{ge}}(f)=0$. Proposition 6.4 implies $R_{B \mid A}^{\mathrm{gl}}(f)=R_{B \mid A}^{\mathrm{ge}}(f)$. 
Remark 7.7 Proposition 7.3 and the proof of Proposition 7.5 show if

$$
\operatorname{dim}(A) \geq 3 \text { and } \operatorname{dim}(B) \geq \operatorname{dim}(A)+2
$$

then $R_{B \mid A}^{\mathrm{KW}}(f)$ is zero if and only if the classical invariants for $A$ and $B$ are zero.

Using these two invariants to define a relative invariant would be analogous to defining the relative invariants in the previous sections as the pair of classical invariants for the spaces $A$ and $B$. This alternate definition would satisfy the requirements of the introduction for a fixed point invariant. However, there are several reasons why the corresponding definition in the equivariant case is not acceptable. The definitions in the previous sections were chosen because they are consistent with the choices in Ponto [20].

\section{Relative sections}

In this section we generalize the result due to Klein and Williams [14] on sections of fibrations to relative fibrations.

If the dimension of $B$ is $2 n$ and the fibration $p: E \rightarrow B$ is $(n+1)-$ connected, it is shown in [14] that the two sections

$$
\sigma_{1}, \sigma_{2}: B \rightarrow S_{B} E
$$

are homotopic over $B$ if and only there is a section of $p$. We will generalize this result to relative sections.

If $A \subset B$ let $E_{A}$ be a subspace of $E_{B}$ such that $p\left(E_{A}\right) \subset A$. Let $S_{A, B} E_{A}$ be

$$
B \times\{0\} \cup E_{A} \times I \cup A \times\{1\} .
$$

Let $\left[\left(S_{B}^{0}, A \amalg B\right),\left(S_{B} E_{B}, S_{A, B} E_{A}\right)\right]_{B}$ be the relative sectioned fiberwise homotopy classes of maps from $\left(S_{B}^{0}, A \amalg B\right)$ to $\left(S_{B} E_{B}, S_{A, B} E_{A}\right)$.

Definition 8.1 Let $A \subset B, p: E_{B} \rightarrow B$ be a fibration, and $E_{A} \subset p^{-1}(A)$ such that $E_{A} \rightarrow A$ is a fibration. The relative homotopy Euler class

$$
\chi \in\left[\left(S_{B}^{0}, A \amalg B\right),\left(S_{B} E_{B}, S_{A, B} E_{A}\right)\right]_{B}
$$

is $\sigma_{1} \amalg \sigma_{2}: S_{B}^{0} \rightarrow S_{B} E_{B}$.

Proposition 8.2 If $\left(E_{B}, E_{A}\right) \rightarrow(B, A)$ admits a relative section $\widetilde{\varsigma}$ then $\chi$ is trivial.

Conversely, assume $p: E_{A} \rightarrow A$ is $(m+1)$-connected, $A$ is a $2 m$-dimensional $C W$ complex, $p: E_{B} \rightarrow B$ is $(n+1)$-connected and $(B, A)$ is a relative $2 n$-dimensional $C W$-complex. If $\chi$ is trivial then $p$ has a relative section. 
Before we prove this proposition we recall a preliminary lemma.

Lemma 8.3 [14, Proposition 3.1] Let $p: E \rightarrow B$ be a $(j+1)$-connected fibration and $P$ be the homotopy pullback

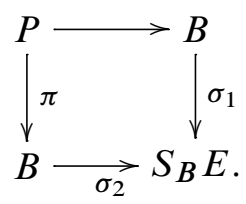

A fiberwise homotopy from $\sigma_{1}$ to $\sigma_{2}$ defines a $2 j$-equivalence $q: E \rightarrow P$.

Note that $\pi q=p$.

Proof of Proposition 8.2 If there is a relative section $\widetilde{\varsigma}$ then the homotopy

$$
H:\left(S_{B}^{0}, A \amalg B\right) \times I \rightarrow\left(S_{B} E_{B}, S_{A, B} E_{A}\right)
$$

defined by $H(b, t)=(\widetilde{\varsigma}(b), t)$ shows $\chi$ is trivial.

If $\chi$ is trivial there is a relative fiberwise homotopy

$$
K:\left(S_{B}^{0}, A \amalg B\right) \times I \rightarrow\left(S_{B} E_{B}, S_{A, B} E_{A}\right)
$$

from $\sigma_{2}$ to $\sigma_{1}$. The restriction of $K$ to $S_{A}^{0}$ defines a homotopy between $\left.\sigma_{1}\right|_{A}: A \rightarrow$ $S_{A} E_{A}$ and $\left.\sigma_{2}\right|_{A}$. Lemma 8.3, Whitehead's theorem, and the homotopy $\left.K\right|_{S_{A}^{0}}$ imply

$$
q_{A *}:\left[A, E_{A}\right] \rightarrow\left[A, P_{A}\right]
$$

is a bijection. The space $P_{A}$ is as in Lemma 8.3.

The restriction $\left.K\right|_{S_{A}^{0}}$ induces a map $h_{A}: A \rightarrow P_{A}$ such that $\pi h_{A}=$ id. Since $q_{A *}$ is a bijection there is a map $k_{A}: A \rightarrow E_{A}$ and a homotopy $J_{A}$ from $q_{A} k_{A}$ to $h_{A}$. Then $p k_{A}=\pi\left(q_{A} k_{A}\right) \simeq \pi h_{A}=\mathrm{id}_{A}$ via the homotopy $\pi\left(J_{A}\right)$. The diagram

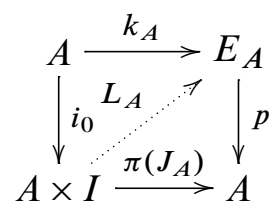

has a lift $L_{A}$, and $p\left(L_{A}(a, 1)\right)=a$. Then $L_{A}(-, 1)$ is a section of $p^{-1}(A) \rightarrow A$ that is contained in $E_{A}$. 
The homotopy $K$ defines a map $h_{B}: B \rightarrow P_{B}$ extending the map $h_{A}$. The space $P_{B}$ is as in Lemma 8.3. The homotopy extension and lifting property implies the dotted maps in the following diagram can be filled in.

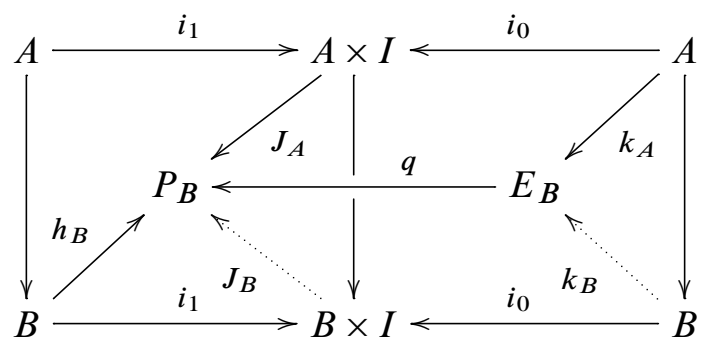

This defines maps $k_{B}$ and $J_{B}$ extending $k_{A}$ and $J_{A}$.

Since the pair $(B, A)$ has the relative homotopy lifting property there is a lift $L_{B}$ in the following diagram.

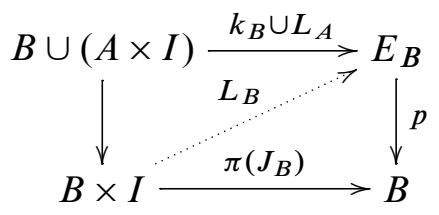

Evaluating at $1, p\left(L_{B}(b, 1)\right)=\pi J_{B}(b, 1)=\pi h_{B}(b)=b$. Since $L_{B}(a, 1) \in E_{A}$ for $a \in A, L_{B}(-, 1)$ is the required section.

Lemma 7.1, Lemma 7.2, and Proposition 8.2 imply $\chi$ is a complete obstruction to determining if a relative fibration has a section. In the examples we are interested in, it is easier to work with invariants defined from $\chi$ than with $\chi$ itself. Under some additional hypotheses, these associated invariants are zero if and only if $\chi$ is zero.

If $A \subset B$, define

$$
C_{B}(B, A):=B \times\{0\} \cup A \times[0,1] \cup B \times\{1\} .
$$

This is an ex-space over $B$ with section given by the inclusion of $B$ into $C_{B}(B, A)$ as $B \times\{0\}$.

In the diagram below the vertical maps are induced by cofiber sequences and so the columns are exact. See Crabb and James [3, Proposition II.2.4]. The horizontal maps 
are forgetful maps. The diagram commutes.

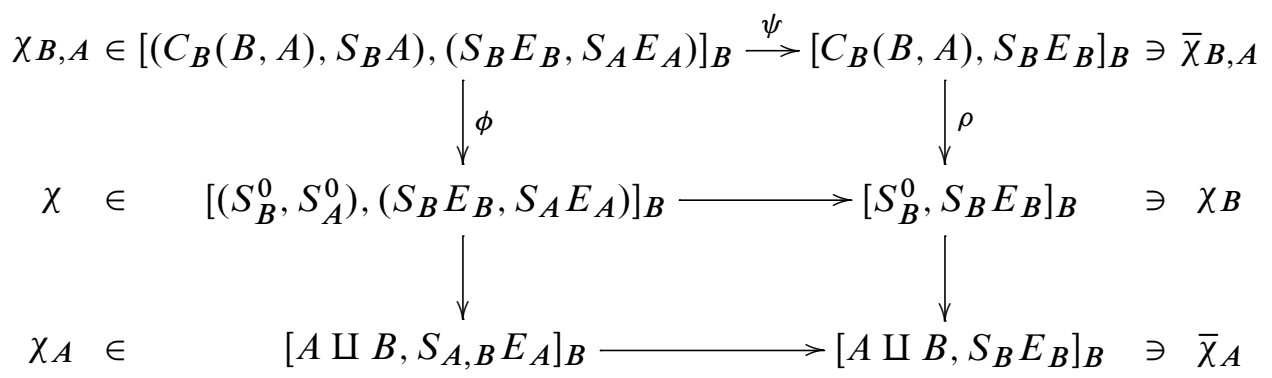

The elements $\chi_{A}, \chi_{B}$, and $\bar{\chi}_{A}$ are the images of $\chi_{\text {. The element }} \chi_{B, A}$ is defined if $\chi_{A}=0$. Then $\chi_{B, A}$ is the preimage of $\chi$. The element $\bar{\chi}_{B, A}$ is defined if $\bar{\chi}_{A}=0$. Then $\bar{\chi}_{B, A}$ is the preimage of $\chi_{B}$.

Lemma 8.4 Suppose $\chi_{A}=0$. If $\bar{\chi}_{B, A}=0$ then $\chi_{B, A}=0$.

Proof Since $\bar{\chi}_{B, A}=0$ there is a fiberwise homotopy

$$
L: C_{B}(B, A) \times I \rightarrow S_{B} E_{B}
$$

such that

$$
\begin{aligned}
& L(b, 1,0)=\sigma_{2}(b) \\
& L(b, 1,1)=\sigma_{1}(b) \\
& L(b, 0, t)=\sigma_{1}(b) \\
& L(a, s, 0)=\chi_{B, A}(a, s) \in S_{A} E_{A} \\
& L(a, s, 1)=\sigma_{1}(a)
\end{aligned}
$$

for all $a \in A, b \in B$, and $s, t \in I$.

Let $J:=(\{0\} \times I) \cup(I \times\{1\}) \cup(\{1\} \times I)$. Define a map

by

$$
\begin{aligned}
\bar{L}: B \times J & \rightarrow S_{B} E_{B} \\
\bar{L}(b, 0, t) & =\sigma_{1}(b) \\
\bar{L}(b, s, 1) & =\sigma_{1}(b) \\
\bar{L}(b, 1, t) & =L(b, 1, t) .
\end{aligned}
$$

The diagram

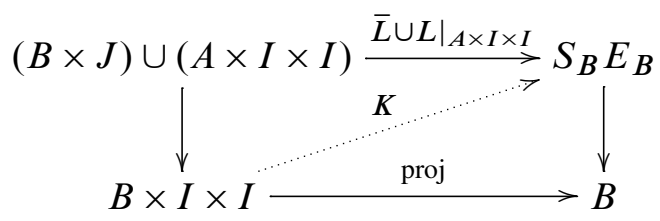

commutes and there is a lift $K$ since $S_{B} E_{B} \rightarrow B$ is a fibration. 
Then $K_{0}:=K(-,-, 0): B \times I \rightarrow S_{B} E_{B}$ satisfies

$$
\begin{aligned}
& K_{0}(b, 0)=K(b, 0,0)=L(b, 0,0)=\sigma_{1}(b) \\
& K_{0}(b, 1)=K(b, 1,0)=L(b, 1,0)=\sigma_{2}(b) \\
& K_{0}(a, s)=K(a, s, 0)=L(a, s, 0) \in S_{A} E_{A} .
\end{aligned}
$$

Define a map

by

$$
\begin{gathered}
\tilde{K}: C_{B}(B, A) \times I \rightarrow S_{B} E_{B} \\
\widetilde{K}(b, 1, t)=K_{0}(b, 1-t) \\
\widetilde{K}(b, 0, t)=\sigma_{1}(b) \\
\tilde{K}(a, s, t)=K_{0}(a, s(1-t)) .
\end{gathered}
$$

$\widetilde{K}$ shows $\chi_{B, A}$ is trivial in $\left[\left(C_{B}(B, A), S_{B} A\right),\left(S_{B} E_{B}, S_{A} E_{A}\right)\right]_{B}$.

Lemma 8.5 If the map $E_{B} \rightarrow B$ is a $(\operatorname{dim}(A)+1)$-equivalence then $\rho$ is injective.

Proof In this proof let $i$ denote the inclusion of $A$ in $B$.

Let $\Sigma_{B}(A \amalg B):=((A \times I) \amalg B) / \sim$ where $(a, 0) \sim i(a) \sim(a, 1)$. Then $\rho$ is part of a long exact sequence

$\left[\Sigma_{B}(A \amalg B), S_{B} E_{B}\right]_{B} \longrightarrow\left[C_{B}(B, A), S_{B} E_{B}\right]_{B}$

$$
\stackrel{\rho}{\longrightarrow}\left[S_{B}^{0}, S_{B} E_{B}\right]_{B} \longrightarrow\left[A \amalg B, S_{B} E_{B}\right]_{B} .
$$

To show that $\rho$ is injective it is enough to show

$$
\left[\Sigma_{B}(A \amalg B), S_{B} E_{B}\right]_{B}
$$

is trivial.

Let $\alpha$ be an element of $\left[\Sigma_{B}(A \amalg B), S_{B} E_{B}\right]_{B}$. Then $\alpha$ defines a map $S^{1} \times A \rightarrow S_{B} E_{B}$ also denoted $\alpha$. This map satisfies $p \alpha(t, a)=i(a)$.

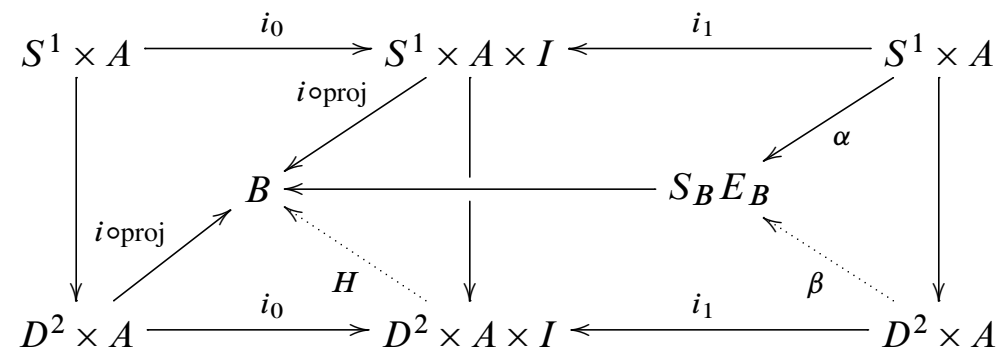


Since $S_{B} E_{B} \rightarrow B$ is a $(\operatorname{dim}(A)+2)$-equivalence, the homotopy extension and lifting property implies there are maps $\beta$ and $H$ that make the diagram commute.

The solid arrow portion of the diagram below commutes.

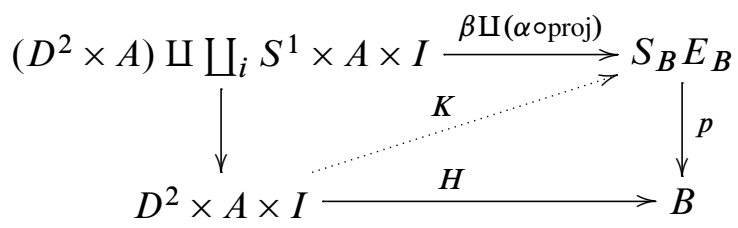

Since $S_{B} E_{B} \rightarrow B$ is a fibration there is a lift $K$ so that the entire diagram commutes. Then

$$
\begin{gathered}
K_{0}:=K(-,-, 0): D^{2} \times A \rightarrow S_{B} E_{B} \\
p K_{0}(v, a)=H(v, a, 0)=i(a) \\
K_{0}(w, a)=\alpha(w, a) .
\end{gathered}
$$

satisfies

and, if $w \in S^{1}$,

Then

$$
K_{0} \amalg \text { id: }\left(\left(D^{2} \times A\right) \amalg B\right) / \sim \rightarrow S_{B} E_{B}
$$

shows $\alpha$ is trivial.

The next proposition is a consequence of Proposition 8.2, Lemma 8.4 and Lemma 8.5.

Proposition 8.6 If $p: E_{A} \rightarrow A$ is $(m+1)$-connected, $A$ is a $2 m$-dimensional $C W-$ complex, $p: E_{B} \rightarrow B$ is $(2 m+1)$-connected and $(B, A)$ is a relative $4 m$-dimensional $C W$-complex $\left(E_{B}, E_{A}\right) \rightarrow(B, A)$ admits a relative section if and only if $\chi_{A}$ and $\chi_{B}$ are both zero.

\section{Obstruction theory}

Obstruction theory provides an alternative to Proposition 7.3. There is a relative section of the fibration

$$
\left(\Gamma_{f *}\left(N\left(i_{B}\right)\right),\left(\Gamma_{\left.f\right|_{A} *}\left(N\left(i_{A}\right)\right)\right) \rightarrow(B, A)\right.
$$

if there is a section of the fibration $\Gamma_{\left.f\right|_{A}}\left(N\left(i_{A}\right)\right) \rightarrow A$ and that section can be extended to a section of the fibration $\Gamma_{f *}\left(N\left(i_{B}\right)\right) \rightarrow B$.

Let $F_{A}$ be the fiber of $\Gamma_{\left.f\right|_{A} *}\left(N\left(i_{A}\right)\right) \rightarrow A$ and $F_{B}$ be the fiber of $\Gamma_{f *}\left(N\left(i_{B}\right)\right) \rightarrow B$. Let $n_{A}=\operatorname{dim}(A)$ and $n_{B}=\operatorname{dim}(B)$.

Using Lemma 7.4 we see there is a single obstruction to finding a section of the map $\Gamma_{\left.f\right|_{A}{ }^{*}}\left(N\left(i_{A}\right)\right) \rightarrow A$. This is a cohomology class $\omega_{A} \in H^{n_{A}}\left(A ; \pi_{n_{A}-1}\left(F_{A}\right)\right)$. The same lemma also shows there is a single obstruction to extending a chosen section over $A$ to 
a section of $\Gamma_{f *}\left(N\left(i_{B}\right)\right) \rightarrow B$. The obstruction to this extension is a cohomology class $\omega_{B, A}$ in $H^{n_{B}}\left(B, A ; \pi_{n_{B}-1}\left(F_{B}\right)\right)$.

The assumptions of Proposition 7.3 significantly simplify the identification of $\omega_{B, A}$. Since $n_{A}+2 \leq n_{B}, H^{n_{B}-1}\left(A ; \pi_{n_{B}-1}\left(F_{B}\right)\right)$ and $H^{n_{B}}\left(A ; \pi_{n_{B}-1}\left(F_{B}\right)\right)$ are both trivial and there is an isomorphism

$$
H^{n_{B}}\left(B, A ; \pi_{n_{B}-1}\left(F_{B}\right)\right) \rightarrow H^{n_{B}}\left(B ; \pi_{n_{B}-1}\left(F_{B}\right)\right) .
$$

The image of $\omega_{B, A}$ is $\omega_{B}$, the obstruction to finding a section of $\Gamma_{f *}\left(N\left(i_{B}\right)\right) \rightarrow B$ with no restriction on $A$.

Recall that a fixed point class of $\left.f\right|_{A}$ determines an element of $\pi_{0}\left(\left.\Lambda^{f}\right|_{A} A\right)$. A choice of base point $*$ in $A$ and path $\tau$ in $A$ from $*$ to $f(*)$ defines a function

$$
\pi: \pi_{1}(A) \rightarrow \pi_{0}\left(\Lambda^{\left.f\right|_{A}} A\right) .
$$

If $A$ is connected this is surjective and two loops $\alpha$ and $\beta$ have the same image under $\pi$ if and only if there is a loop $\gamma$ in $A$ so that $\gamma \beta f_{*}\left(\gamma^{-1}\right) \simeq \alpha$. We will identify a fixed point class with the corresponding element of $\pi_{1} A$ modulo the relation above.

Fadell and Husseini [8, Corollary 3.2] defined isomorphisms $\pi_{n_{A}-1}\left(F_{A}\right) \cong \mathbb{Z} \pi_{1}(A)$ and $\pi_{n_{B}-1}\left(F_{B}\right) \cong \mathbb{Z} \pi_{1}(B)$. They also showed, [8, Theorem 1.2], that $\omega_{A}$ and $\omega_{B}$ have cochain representatives

$$
c_{A}(f)=\sum i\left(\left.f\right|_{A}, \alpha\right) \alpha s \quad \text { and } \quad c_{B}(f)=\sum i(f, \beta) \beta t
$$

where $s$ is a $n_{A}$ simplex of $A$ that contains the base point, $t$ is a $n_{B}$ simplex of $B$ that contains the base point, the first sum is over fixed point classes of $\left.f\right|_{A}$ and the second sum is over fixed point classes of $f$.

Then Corollary 5.8 shows $\omega_{A}$ and $\omega_{B}$ are both zero if and only if $R_{B \mid A}^{\mathrm{ge}}(f)$ is zero. This replaces Proposition 7.5 in the proof of Theorem B.

\section{Other descriptions of $\odot$ in special cases}

These are the proofs omitted from Section 3. Let $\mathscr{A}$ be an EI-category enriched in the category of abelian groups.

Lemma 3.6 If $\mathscr{X}: \mathscr{A} \rightarrow \mathrm{Ch}_{R}$ and $\mathscr{Y}: \mathscr{A}^{\text {op }} \rightarrow \mathrm{Ch}_{R}$ are supported on isomorphisms

$$
\mathscr{X} \odot \mathscr{Y} \cong \bigoplus_{c \in B(\mathscr{A})} \mathscr{X}(c) \otimes_{\mathscr{A}(c, c)} \mathscr{Y}(c) .
$$


Proof We will show that $\oplus \mathscr{X}(c) \otimes_{\mathscr{A}(c, c)} \mathscr{Y}(c)$ satisfies the universal property that defines $\mathscr{X} \odot \mathscr{Y}$.

By definition of $B(\mathscr{A})$, for any object $a$ in $\mathscr{A}$ there is exactly one object

$$
c \in B(\mathscr{A})
$$

such that there is an isomorphism $f: a \rightarrow c$ in $\mathscr{A}$. Define a map

$$
\theta_{a}: \mathscr{X}(a) \otimes_{\mathbb{Z}} \mathscr{Y}(a) \rightarrow \mathscr{X}(c) \otimes_{\mathscr{A}(c, c)} \mathscr{Y}(c)
$$

as the composite of

$$
\mathscr{X}(f) \otimes \mathscr{Y}\left(f^{-1}\right): \mathscr{X}(a) \otimes_{\mathbb{Z}} \mathscr{Y}(a) \rightarrow \mathscr{X}(c) \otimes_{\mathbb{Z}} \mathscr{Y}(c)
$$

with the quotient map

$$
\mathscr{X}(c) \otimes_{\mathbb{Z}} \mathscr{Y}(c) \rightarrow \mathscr{L}(c) \otimes_{\mathscr{A}(c, c)} \mathscr{Y}(c) .
$$

If $g$ is another isomorphism in $\mathscr{A}$ from $a$ to $c$, then $\left(\mathscr{L}(f)(A), \mathscr{Y}\left(f^{-1}\right)(B)\right)$ is identified with $\left(\mathscr{L}(g)(A), \mathscr{Y}\left(g^{-1}\right)(B)\right)$ and the map $\theta_{a}$ is well defined. Let

$$
\theta: \bigoplus_{a \in \operatorname{ob}(\mathscr{A})} \mathscr{X}(a) \otimes_{\mathbb{Z}} \mathscr{Y}(a) \rightarrow \bigoplus_{c \in B(\mathscr{A})} \mathscr{X}(c) \otimes_{\mathscr{A}(c, c)} \mathscr{Y}(c)
$$

be the sum of the maps $\theta_{a}$.

If $(A, f, B) \in \mathscr{X}(a) \otimes_{\mathbb{Z}} \mathscr{A}(a, b) \otimes_{\mathbb{Z}} \mathscr{Y}(b)$ the images of this element in

$$
\bigoplus_{a \in \mathrm{ob}(\mathscr{A})} \mathscr{L}(a) \otimes_{\mathbb{Z}} \mathscr{Y}(a)
$$

are $(A, \mathscr{Y}(f)(B))$ and $(\mathscr{X}(f)(A), B)$. The images of these elements are identified under $\theta$.

Let

$$
\phi: \bigoplus_{a \in \mathrm{ob} \mathscr{A}} \mathscr{X}(a) \otimes_{\mathbb{Z}} \mathscr{Y}(a) \rightarrow M
$$

be a map that coequalizes the two maps from $\bigoplus_{a, b \in \mathrm{ob} \mathscr{A}} \mathscr{X}(a) \otimes_{\mathbb{Z}} \mathscr{A}(a, b) \otimes_{\mathbb{Z}} \mathscr{Y}(b)$ to $\bigoplus_{a \in \mathrm{ob} \mathscr{A}} \mathscr{X}(a) \otimes_{\mathbb{Z}} \mathscr{Y}(a)$. Define a map

$$
\psi: \bigoplus_{c \in B(\mathscr{A})} \mathscr{X}(c) \otimes_{\mathscr{A}(c, c)} \mathscr{Y}(c) \rightarrow M
$$

by choosing lifts of elements in $\mathscr{X}(c) \otimes_{\mathscr{A}(c, c)} \mathscr{Y}(c)$ to elements of

$$
\mathscr{X}(c) \otimes_{\mathbb{Z}} \mathscr{Y}(c) .
$$

Since $\phi$ coequalizes, the choices do not matter and $\psi$ is unique. 
Lemma 3.7 Let $\mathscr{X}$ and $\mathscr{y}$ satisfy the conditions of Lemma 3.6. If $\mathscr{X}(c)$ is dualizable as a $\mathscr{A}(c, c)$-module with dual $\mathscr{Y}(c)$ for each $c \in B(\mathscr{A})$ then $\mathscr{X}$ is dualizable with dual $\mathrm{Y}$.

Proof If $\mathscr{L}(c)$ is dualizable as an $\mathscr{A}(c, c)$-module with dual $\mathscr{Y}(c)$ then there is a map of chain complexes of abelian groups

$$
\eta_{c}: \mathbb{Z} \rightarrow \mathscr{L}(c) \odot \mathscr{Y}(c)
$$

and a map of chain complexes of $\mathscr{A}(c, c)$-bimodules

$$
\epsilon_{c}: \mathscr{y}(c) \odot \mathscr{X}(c) \rightarrow \mathscr{A}(c, c)
$$

for each $c \in B(\mathscr{A})$.

Let $\eta: \mathbb{Z} \rightarrow \mathscr{X} \odot \mathscr{y}$ be the composite

$$
\mathbb{Z} \stackrel{\Delta}{\rightarrow} \bigoplus_{B(\mathscr{A})} \mathbb{Z} \stackrel{\oplus \eta_{c}}{\rightarrow} \bigoplus_{B(\mathscr{A})} \mathscr{X}(c) \otimes_{\mathscr{A}(c, c)} \mathscr{Y}(c) \cong \mathscr{X} \odot \mathscr{Y}
$$

where $\triangle: \mathbb{Z} \rightarrow \bigoplus_{B(\&)} \mathbb{Z}$ is the map that takes 1 to $(1,1, \ldots, 1)$.

Let $a$ and $b$ be isomorphic objects of $\mathscr{A}$. Let $c$ be an object of $B(\mathscr{A})$ that is isomorphic to $a$, let $h$ be an isomorphism in $\mathscr{A}$ from $a$ to $c$ and let $g$ be an isomorphism from $b$ to $c$. Then $\epsilon_{a, b}$ is the following composite:

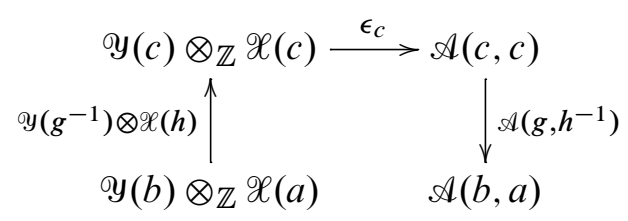

If $a$ and $b$ are not isomorphic in $\mathscr{A} \epsilon_{a, b}$ is zero. Since $c$ is unique and the maps $\epsilon_{c}$ are maps of $\mathscr{A}(c, c)$-bimodules, $\epsilon$ is a natural transformation. This also implies that $\epsilon$ is independent of the choice of $g$ and $h$.

Let $\eta_{c}(1)=\sum_{i} e_{c, i} \otimes f_{c, i}$ for each $c \in B(\mathscr{A})$. If $x \in \mathscr{L}(a)$ the value of the composite

$$
\mathscr{X}(a) \cong \mathbb{Z} \otimes \mathscr{X}(a) \stackrel{\eta \otimes 1}{\rightarrow} \mathscr{X} \odot \mathscr{Y} \otimes \mathscr{X}(a) \stackrel{1 \odot \epsilon}{\rightarrow} \mathscr{X} \odot \mathscr{A}(-, a) \cong \mathscr{L}(a)
$$

applied to $x$ is

$$
\sum_{c \in B(A)} \sum_{i} \mathscr{X}\left(\epsilon\left(f_{c, i}, x\right)\right)\left(e_{c, i}\right)
$$


The only nonzero terms in this sum are those where there is an isomorphism $h$ from $x$ to $c$. By definition, $\epsilon\left(f_{c, i}, x\right)=h^{-1} \epsilon_{c}\left(f_{c, i}, \mathscr{X}(h)(x)\right)$ and

$$
\begin{aligned}
\sum_{i} \mathscr{X}\left(\epsilon\left(f_{c, i}, x\right)\right)\left(e_{c, i}\right) & =\mathscr{X}\left(h^{-1}\right) \sum_{i} \mathscr{X}\left(\epsilon_{c}\left(f_{c, i}, \mathscr{L}(h)(x)\right)\right)\left(e_{c, i}\right) \\
& =\mathscr{X}\left(h^{-1}\right) \mathscr{X}(h)(x) \\
& =x .
\end{aligned}
$$

The other diagram is similar.

\section{References}

[1] C Bowszyc, Fixed point theorems for the pairs of spaces, Bull. Acad. Polon. Sci. Sér. Sci. Math. Astronom. Phys. 16 (1968) 845-850 MR0246290

[2] R F Brown, The Lefschetz fixed point theorem, Scott, Foresman and Co., Glenview, Ill.-London (1971) MR0283793

[3] M Crabb, I James, Fibrewise homotopy theory, Springer Monogr. in Math., Springer, London (1998) MR1646248

[4] T tom Dieck, Transformation groups, de Gruyter Studies in Math. 8, de Gruyter, Berlin (1987) MR889050

[5] A Dold, The fixed point transfer of fibre-preserving maps, Math. Z. 148 (1976) 215-244 MR0433440

[6] A Dold, Lectures on algebraic topology, Classics in Math., Springer, Berlin (1995) MR1335915 Reprint of the 1972 edition

[7] A Dold, D Puppe, Duality, trace, and transfer, from: "Proceedings of the International Conference on Geometric Topology (Warsaw, 1978)", (K Borsuk, A Kirkor, editors), PWN, Warsaw (1980) 81-102 MR656721

[8] E Fadell, S Husseini, Fixed point theory for non-simply-connected manifolds, Topology 20 (1981) 53-92 MR592570

[9] S Husseini, Generalized Lefschetz numbers, Trans. Amer. Math. Soc. 272 (1982) 247274 MR656489

[10] I M James, Fibrewise complexes, from: "Algebraic topology: new trends in localization and periodicity (Sant Feliu de Guíxols, 1994)", (C Broto, C Casacuberta, G Mislin, editors), Progr. Math. 136, Birkhäuser, Basel (1996) 193-199 MR1397730

[11] J Jezierski, A modification of the relative Nielsen number of $H$ Schirmer, Topology Appl. 62 (1995) 45-63 MR1318427

[12] B J Jiang, Lectures on Nielsen fixed point theory, Contemporary Math. 14, Amer. Math. Soc. (1983) MR685755 
[13] B J Jiang, X Zhao, H Zheng, On fixed points of stratified maps, J. Fixed Point Theory Appl. 2 (2007) 225-240 MR2372986

[14] J R Klein, B Williams, Homotopical intersection theory. I, Geom. Topol. 11 (2007) 939-977 MR2326939

[15] J R Klein, B Williams, Homotopical intersection theory. II. Equivariance, Math. Z. 264 (2010) 849-880 MR2593297

[16] L G Lewis, Jr, J P May, M Steinberger, J E McClure, Equivariant stable homotopy theory, Lecture Notes in Math. 1213, Springer, Berlin (1986) MR866482 With contributions by $\mathrm{J}$ E McClure

[17] W Lück, Transformation groups and algebraic $K$-theory, Lecture Notes in Math. 1408, Springer, Berlin (1989) MR1027600 Mathematica Gottingensis

[18] J P May, J Sigurdsson, Parametrized homotopy theory, Math. Surveys and Monogr. 132, Amer. Math. Soc. (2006) MR2271789

[19] B Norton-Odenthal, P Wong, A relative generalized Lefschetz number, Topology Appl. 56 (1994) 141-157 MR1266139

[20] K Ponto, Equivariant fixed point theory arXiv:0910:1274

[21] K Ponto, Fixed point theory and trace for bicategories, Astérisque (2010) xii+102 MR2741967

[22] K Ponto, M Shulman, Shadows and traces in bicategories arXiv:0910.1306

[23] K Reidemeister, Automorphismen von Homotopiekettenringen, Math. Ann. 112 (1936) 586-593 MR1513064

[24] H Schirmer, A relative Nielsen number, Pacific J. Math. 122 (1986) 459-473 MR831126

[25] H Schirmer, On the location of fixed points on pairs of spaces, Topology Appl. 30 (1988) 253-266 MR972697

[26] J Stallings, Centerless groups-an algebraic formulation of Gottlieb's theorem, Topology 4 (1965) 129-134 MR0202807

[27] A Strøm, Note on cofibrations, Math. Scand. 19 (1966) 11-14 MR0211403

[28] F Wecken, Fixpunktklassen. II. Homotopieinvarianten der Fixpunkttheorie, Math. Ann. 118 (1941) 216-234 MR0010280

[29] X Zhao, Minimal fixed point sets of relative maps, Fund. Math. 162 (1999) 163-180 MR1734915

[30] X Zhao, On minimal fixed point numbers of relative maps, Topology Appl. 112 (2001) 41-70 MR1815271

[31] X Zhao, Relative Nielsen theory, from: "Handbook of topological fixed point theory", (R F Brown, M Furi, L Górniewicz, B J Jiang, editors), Springer, Dordrecht (2005) 659-684 MR2171120 
[32] X Zhao, A relative Reidemeister trace, JP J. Fixed Point Theory Appl. 1 (2006) 65-84 MR2351780

Department of Mathematics, University of Kentucky

719 Patterson Office Tower, Lexington KY 40506, USA

kate.ponto@uky.edu

http://www.ms . uky.edu/ kate

Received: 1 November 2009 Revised: 2 December 2010 